\title{
Symbiotic Organism Search Algorithm for Power Loss Minimization in Radial Distribution Systems by Network Reconfiguration and Distributed Generation Placement
}

\author{
Tung Tran The, ${ }^{1,2}$ Sy Nguyen Quoc, ${ }^{3}$ and Dieu Vo Ngoc $\mathbb{( D}^{1,2}$ \\ ${ }^{1}$ Department of Power Systems, Ho Chi Minh City University of Technology (HCMUT), 268 Ly Thuong Kiet Street, District 10, \\ Ho Chi Minh City, Vietnam \\ ${ }^{2}$ Vietnam National University Ho Chi Minh City, Linh Trung Ward, Thu Duc District, Ho Chi Minh City, Vietnam \\ ${ }^{3}$ Tra Vinh Vocational College, Vinh Yen Hamlet, Long Duc Commune, Tra Vinh, Tra Vinh Province, Vietnam
}

Correspondence should be addressed to Dieu Vo Ngoc; vndieu@gmail.com

Received 29 February 2020; Revised 21 April 2020; Accepted 19 May 2020; Published 22 June 2020

Academic Editor: Yang Li

Copyright (C) 2020 Tung Tran The et al. This is an open access article distributed under the Creative Commons Attribution License, which permits unrestricted use, distribution, and reproduction in any medium, provided the original work is properly cited.

\begin{abstract}
This paper proposes the Symbiotic Organism Search (SOS) algorithm to find the optimal network configuration and the placement of distributed generation (DG) units that minimize the real power loss in radial distribution networks. The proposed algorithm simulates symbiotic relationships such as mutualism, commensalism, and parasitism for solving the optimization problems. In the optimization process, the reconfiguration problem produces a large number of infeasible network configurations. To reduce these infeasible individuals and ensure the radial topology of the network, the graph theory was applied during the power flow. The implementation of the proposed SOS algorithm was carried out on 33-bus, 69-bus, 84-bus, and 119-bus distribution networks considering seven different scenarios. Simulation results and performance comparison with other optimization methods showed that the SOS-based approach was very effective in solving the network reconfiguration and DG placement problems, especially for complex and large-scale distribution networks.
\end{abstract}

\section{Introduction}

The distribution network is an important part of the power system. It delivers electricity from the transmission system to customers. The power loss in distribution networks is high due to low voltage and high current compared to the transmission system [1]. In order to reduce the power loss in distribution networks, several methods are used such as network reconfiguration, capacitors installation, increasing size of conductors, and changing transformer taps. Among these methods, network reconfiguration requires less investment cost for electric utilities since it utilizes the available resources of existing grids, while other methods require additional cost for the installation of capacitors, conductors, and tap changing transformers. Generally, distribution networks are operated in the radial structure for effective protection coordination and to reduce the fault level. There are two types of switches including sectionalizing switches (normally closed (NC)) and tie-line switches (normally opened (NO)) used in distribution networks. The distribution network reconfiguration (DNR) is performed by changing the status of those NC/NO switches in the network to form a new radial structure so as the power loss is minimized while satisfying all the operating constraints [2].

Researchers have suggested different approaches such as heuristic methods and metaheuristic methods to solve the DNR problem over the past few decades. Merlin and Back [3] were first to propose a method for this problem to reduce the power system loss. The DNR problem was formulated as an integer mixed nonlinear optimization problem and solved by using a discrete branch-and-bound technique. In [4], the branch exchange scheme was used to determine open/closed states of switches in distribution networks for loss reduction and load demand balance. In [5], a new 
heuristic approach of branch exchange was developed based on the direction of the branch power flows to reduce the active power loss of distribution networks. In [6], a network topology approach was used to describe the formulation of the DNR problem. The single loop optimization technique was implemented to determine the network configuration. In which, a heuristic switch plan was developed for the DNR problem from initial configuration to optimal configuration. In general, heuristic methods are easy to implement and provide fast computational time and good solution for small-scale systems. However, these methods are not really effective for dealing with large-scale systems. Therefore, this motivates the exploration of new effective methods to cope with the large-scale DNR problem.

Metaheuristic methods inspired by nature have proved their capacity to deal with different optimization problems in engineering fields. They have a powerful search ability to find near-optimal or optimal solution and are applicable to large-scale networks. In the field of power system engineering, the DNR problem was successfully solved by Genetic Algorithm (GA). In [7], Zhu proposed a refined GA for the DNR problem to minimize the system power loss. Mendoza et al. [8] used GA with a restricted population and new operators of accentuated crossover and directed mutation for minimal loss reconfiguration problem. Torres et al. [9] improved a GA using the edge window decoder encoding technique for solving the DNR problem with minimal losses. Another well-known metaheuristic optimization method is Particle Swarm Optimization (PSO), which is widely applied for the DNR problem. In [10], Wu and Tsai proposed the PSO with integer coded to find the switch operation schemes for the DNR problem. Gupta et al. [11] used an adaptive PSO to reconfigure the radial distribution systems for minimizing the real power loss. Li and Xuefeng [12] proposed the Niche Binary PSO (NBPSO) algorithm to overcome the defect of PSO prematurity for the DNR problem. Pegado et al. [13] presented an Improved Selective Binary PSO (IS-BPSO) for solving the DNR problem with the aim of reducing power losses. In [14, 15], an Improved Tabu Search (ITS) algorithm and Harmony Search Algorithm (HSA) were, respectively, successfully applied to solve the DNR problem for power loss reduction in large-scale distribution networks. In [16], an ordinal optimization (OO) approach was presented to solve the DNR problem, which can reduce the computational time for the DNR problem. Other optimization techniques such as Cuckoo Search Algorithm (CSA) [17], Fireworks Algorithm (FWA) [18], and Adaptive Shuffled Frogs Leaping Algorithm (ASFLA) [19] were also applied to the DNR problem for power loss minimization and voltage profile improvement. In [20], the DNR problem was formulated with multiobjective of real power loss reduction, load balancing among the branches, load balancing among the feeders, number of switching operations, and node voltage deviation and solved by Runner-Root Algorithm combined with fuzzy logic technique.

In recent years, distributed generation (DG) is rapidly deployed in distribution networks due to the depletion of fossil fuels, environmental concerns, and electricity deregulation. The proper installation of DG units reduces power loss and improves overall voltage of the networks. Therefore, the DNR problem should be considered in the presence of DG. Several studies have proposed the combination of network reconfiguration and optimal DG placement to improve the performance of distribution networks. In [21, 22], Harmony Search Algorithm (HSA) and Fireworks Algorithm (FWA) were, respectively, proposed to solve the DNR problem together with DG placement in 33- and 69bus radial distribution networks (RDNs). The objective was to reduce the real power loss and improve voltage profile of the networks. Rao et al. [21] used the loss sensitivity factor (LSF) method to preidentify the optimal candidate locations for DG installation, whereas the study [22] used the voltage stability index (VSI). In these studies, the DG locations were preidentified by the LSF and VSI methods, and the HSA and FWA methods only optimized the sizes of DG units. Therefore, the variables of locations and sizes of DG units were not simultaneously optimized with the DNR problem. In [23], an Adaptive Cuckoo Search Algorithm (ACSA) was implemented for simultaneously solving the DNR and the optimal DG placement (ODGP) problems with the same objective in [21]. The graph theory was used to reduce infeasible network configurations and check the radial network constraint. In [24], an optimization algorithm based on the electromagnetism-like mechanism (ELM) was applied to simultaneously optimize the configuration of distributions network and the placement of the DG units. In [25], a hybrid technique of two algorithms, Grey Wolf Optimizer (GWO) and PSO, was also presented to solve the DNR problem while considering DG. Murty and Kumar [26] proposed a multiobjective optimization problem for optimal DG placement and network reconfiguration considering the uncertainties of renewable-based DGs and load. The proposed problem was solved by a combination of Gravitational Search Algorithm (GSA) and General Algebraic Modelling System (GAMS).

Based on the literature review, most of the researchers [3-21] only focused on the DNR problem and ignored the integration of DG units. A few studies [22-27] have discussed a combination of network reconfiguration and optimal DG placement. These studies showed that the combination of the DNR and ODGP problems effectively reduced the power loss and improved overall voltage of distribution networks; however, the large-scale systems did not consider these studies. Moreover, most of the studies used metaheuristic methods to solve the formulated optimization problem. Although metaheuristic methods possess a powerful search ability, they may not reach optimal solution and consume long computational time for large-scale systems. Therefore, there is always a need to validate new optimization techniques for solving complex optimization problems. This study proposes a Symbiotic Organisms Search (SOS) algorithm to solve the DNR problem along with the optimal DG placement, especially in large-scale systems.

The SOS algorithm was proposed by Cheng and Prayogo for solving numerical optimization and engineering design problems [27]. This algorithm simulates symbiotic relationships including mutualism, commensalism, and 
parasitism in nature. The advantage of SOS is its simple structure with only two controllable parameters (i.e., Eco_size (population size) and the number of iterations) that makes it easy to implement. For this reason, the SOS algorithm has recently become a popular choice to apply to different optimization problems such as project scheduling [28], vehicle routing [29], structural design [30, 31], multiobjective constrained optimization problem [32], optimization problems in power system [33-36], optimal scheduling of tasks on cloud and time-cost-labor utilization trade-off problem [37], and the reconfiguration problem of distribution networks with distributed generations [38]. In this study, the SOS algorithm was implemented to solve the DNR problem together with the ODGP problem in distribution networks. The objective function of this study is to minimize real power loss that is subject to a set of operating system constraints such as power balance, bus voltage limits, feeder capacity limits, DG capacity limits, and radial configuration constraint. For dealing with the problem, the proposed SOS method is implemented with the help of the graph theory. According to graph theory, a distribution network can be represented with a graph that includes a set of nodes and a set of lines. The graph theory can be considered as a problem of finding an optimal tree of the graph that ensures the radial topology of a distribution network. The graph theory helps the SOS method to reduce infeasible network configurations and check the radial constraint during the optimization process. The LFS method was also used to predetermine the locations of DG units. The proposed algorithm was tested on different radial distribution networks with seven different scenarios. The results obtained from the SOS method were compared to those from other optimization methods available in the literature to evaluate the effectiveness of the proposed method. follows:

The contributions of this study can be summarized as

(i) A Symbiotic Organism Search (SOS) algorithm and the graph theory were effectively implemented for solving the reconfiguration problem with distributed generations

(ii) Seven different scenarios of the reconfiguration problem with distributed generations were considered to evaluate the effectiveness of the proposed SOS method

(iii) The SOS method was tested on different large-scale distribution networks including 33-bus, 69-bus, 84bus, and 119-bus

(iv) The result comparisons have shown that the SOS method is superior to other methods in terms of the obtained optimal solution quality

The rest of this paper is outlined as follows: Section 2 presents mathematical formulation of the DNR and ODGA problems. Section 3 describes the methodology for solving the DNR problem. Section 4 and Section 5, respectively, introduce the LSF method and the proposed SOS method in detail. Section 6 describes the step-by-step implementation of SOS to the formulated problem, which is followed by the simulation results in Section 7. Finally, the conclusion is given in Section 8 .

\section{Problem Formulation}

The main objective of this study is to minimize the real power loss in a distribution work via the network reconfiguration and optimal placement of DG units while satisfying all the system operating constraints. Mathematically, the objective function is formulated as follows:

$$
\mathrm{OF}=\min \left(P_{L}\right)
$$

where

$$
P_{L}=\sum_{i=1}^{N_{b r}} R_{i} \times \frac{P_{i}^{2}+Q_{i}^{2}}{V_{i}^{2}}
$$

where OF denotes the objective function; $P_{L}$ is the real power loss; $R_{i}$ is the resistance of the $i^{\text {th }}$ branch; $P_{i}$ and $Q_{i}$ are the active and reactive powers load at $i$ th bus, respectively; $V_{i}$ is the voltage magnitude at $i$ th bus; and $N_{b r}$ is the total number of branches in a distribution network.

The objective function in (1) is subject to the following constraints.

2.1. Real and Reactive Power Balance. The active and reactive power in the network should be balanced as follows:

$$
\begin{aligned}
P_{\text {slack }}+\sum_{i=1}^{N_{D G}} P_{D G, i} & =\sum_{j=1}^{N_{b}} P_{D, j}+\sum_{k=1}^{N_{b r}} P_{L, k}, \\
Q_{\text {slack }}+\sum_{i=1}^{N_{D G}} Q_{D G, i} & =\sum_{j=1}^{N_{b}} Q_{D, j}+\sum_{k=1}^{N_{b r}} Q_{L, k},
\end{aligned}
$$

where $N_{D G}$ is the total number of DG units to be integrated; $N_{b}$ is the total number of buses in a distribution network; $P_{\text {slack }}$ and $Q_{\text {slack }}$ are the active and reactive powers supplied from the slack bus, respectively; $P_{D G, i}$ and $Q_{D G, i}$ are the active and reactive power outputs of the $i$ th DG unit, respectively; $P_{D, j}$ and $Q_{D, j}$ are the active and reactive power load demands at the $j$ th bus, respectively; and $P_{L, k}$ and $Q_{L, k}$ are the active and reactive power losses in the $k$ th branch, respectively.

2.2. Bus Voltage Limits. The bus voltages should be restricted within their minimum and maximum limits as below:

$$
V_{\min , i} \leq V_{i} \leq V_{\max , i} ; \quad i=1, \ldots, N_{b},
$$

where $V_{\min , i}$ and $V_{\max , i}$ are the minimum and maximum voltage of the $i$ th bus, respectively.

2.3. Feeder Capacity Limits. The current flow in branches should not exceed their maximum limit described by

$$
0 \leq I_{i} \leq I_{\max , i} ; \quad i=1, \ldots, N_{b r},
$$

where $I_{i}$ is the current passing through $i$ th branch and $I_{\max , i}$ is the maximum current allowed to flow through that branch. 
2.4. DG Capacity Limits. The capacity of DG units should not exceed their limits:

$$
P_{D G \min , i} \leq P_{D G, i} \leq P_{D G \max , i} ; \quad i=1,2, \ldots, N_{D G},
$$

where $P_{D G \min , i}$ and $P_{D G \max , i}$ are the minimum and maximum sizes of the $i$ th DG unit, respectively.

2.5. Radial Configuration Constraint. The distribution network must ensure its radial configuration and serve all loads after reconfiguration.

\section{Methodology for Solving the DNR Problem}

3.1. Fundamental Loop. In population-based metaheuristic methods, the initial population is randomly generated. When a metaheuristic method is applied to deal with the DNR problem, the generation of population creates a large number of infeasible network configurations that violate radial topology constraint. To reduce infeasible solutions, this study performed the fundamental loop (FL) using graph theory [39]. To determine FLs, an incidence matrix $A$, which represents the graph of a distribution network, is created. Matrix $A$ consists of $N_{b r}$ rows and $N_{b}$ columns. The elements of matrix $A$ are determined as below [40]:

$$
A_{i j}= \begin{cases}1, & \text { if } i^{\text {th }} \text { branch is directed away from } j^{\text {th }} \text { node, } \\ -1, & \text { if } i^{\text {th }} \text { branch is directed toward from } j^{\text {th }} \text { node }, \\ 0, & \text { otherwise. }\end{cases}
$$

The number of FLs of a distribution network is equal to the number of tie-line switches [39]. For finding the first FL, an open branch, which contains the first tie-switch, is added to connection matrix $A$. Sum of the absolute value of each element in each column of matrix $A$ is calculated. Nodes, which have the sum result as 1, are determined. Branches connected to those nodes are eliminated. This procedure is repeated until the sum result is no longer equal to 1 . The numbers of remaining branches can be stored in a vector that represents a first FL. The remaining FLs are determined as the same procedure of the first FL [39]. Figure 1 shows the graph of an IEEE 16-bus distribution network, which has three tie-switches: 14-15-16. An example of finding the FLs of this system is described by the following steps:

(i) Step 1: add the branch contained tie-switch 14 to the connection matrix $A$.

(ii) Step 2: in each column of matrix $A$, calculate the sum of the absolute value of each element. The nodes, which have the sum result as 1 , are nodes 3,8 , 9,10 , and 12 . The branches connected to these nodes are branches $2,6,12,13$, and 8 . These branches are removed.

(iii) Step 3: do the same as step 2, and branch 5 is removed.

(iv) Step 4: do the same as step 2, and branch 4 is removed.

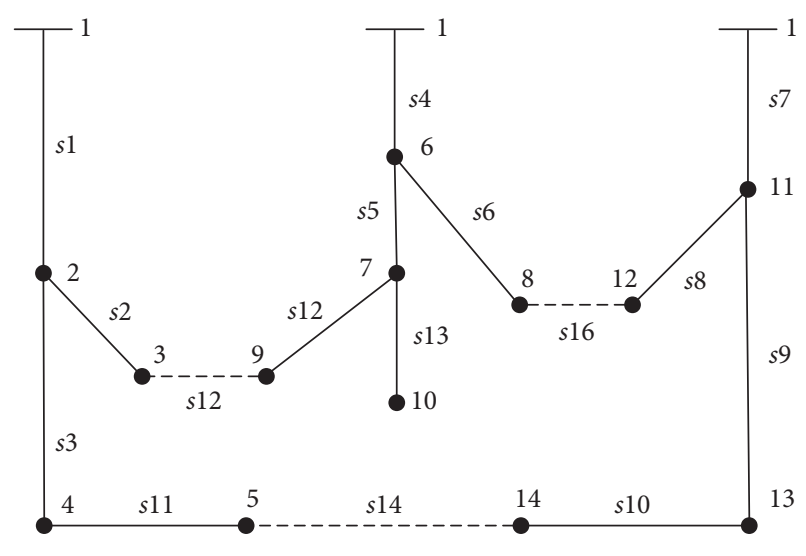

Figure 1: IEEE 16-bus system.

(v) Step 5: at this step, in each column of matrix $A$, the sum of the absolute value of each element is no longer equal to 1 . Therefore, the first FL is determined as branches $1,3,7,9,10,11$, and 14 .

The procedure to determine the first FL of the IEEE 16bus system is depicted in Figure 2. Similar to the determination of first FL, the second FL is determined by adding the branch contained tie-switch 15 . The result of the second FL is branches $1,2,4,5,12$, and 15 . Similarly, the third FL with branches $4,6,7,8$, and 16 is obtained.

3.2. Radial Constraint Checking. After the FLs are determined, the network configuration must be checked for radial topology. The steps for checking the radial structure of a distribution network are described as follows [40]:

(i) Step 1: create an incidence matrix $A$ (as represented in Section 4.1) for each configuration of the network.

(ii) Step 2: remove the first column of matrix $A$ corresponding to the reference node.

(iii) Step 3: remove the rows of matrix $A$ corresponding to tie-switches.

(iv) Step 4: check the conditions. The rest of matrix $A$ is a square matrix, and its determinant is 1 or -1 .

(v) Step 5: if both conditions above are satisfied, the network configuration is a radial topology.

\section{Loss Sensitive Factor}

For the DNR problem considering the allocation of DG units, this study applies the LSF method [21] to determine the candidate bus locations to install DG units in the network before solving the reconfiguration problem.

Considering a simple distribution network with an impedance of $R_{k}+j X_{k}$ and a load of $P_{\text {eff }}+j Q_{\text {eff }}$ connecting between two nodes $p$ and $q$, as shown Figure 3, the real loss power on $k$ th line is calculated as follows:

$$
P_{\text {lineloss }, k}=\frac{\left(P_{\text {eff }}^{2}[q]+Q_{\text {eff }}^{2}[q]\right) \times R_{k}}{(V[q])^{2}} .
$$




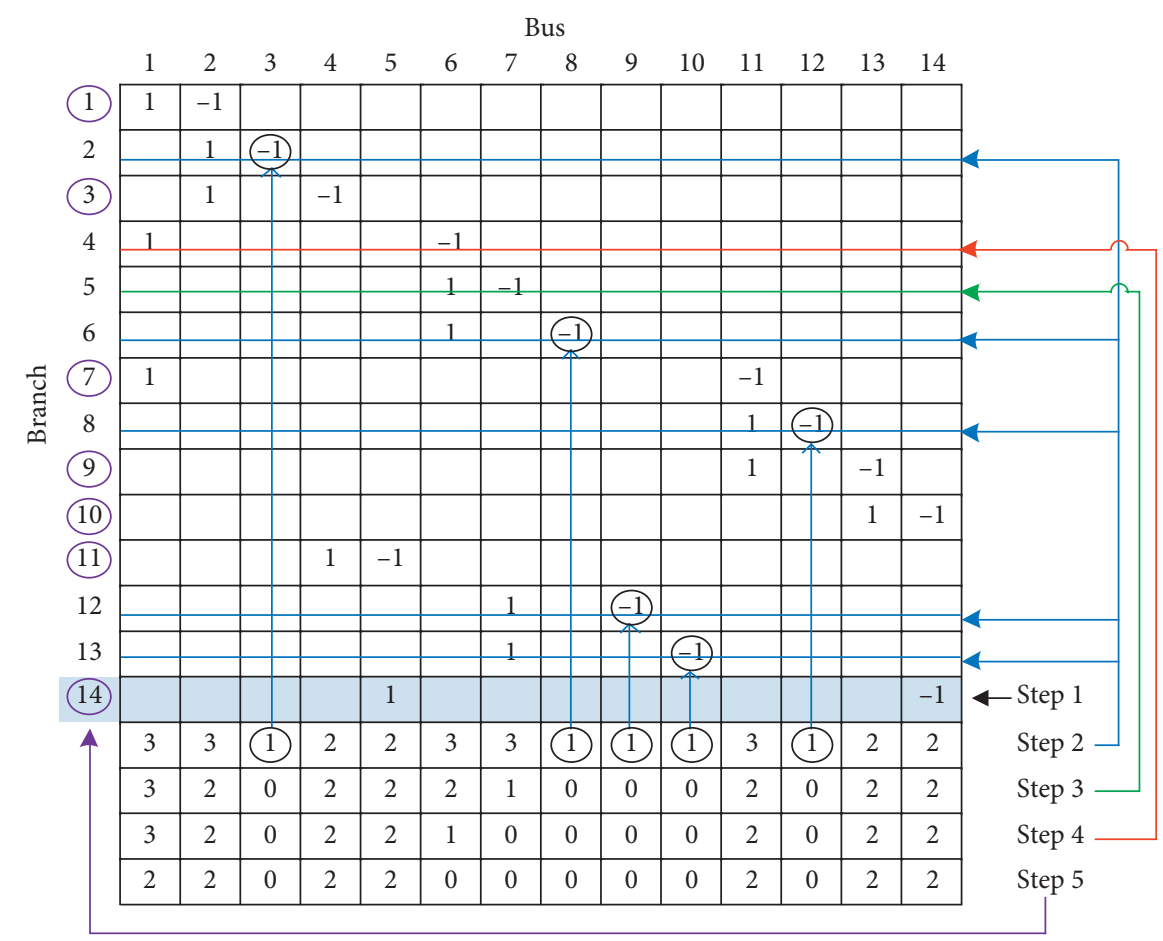

FIgure 2: Determination of the first FL of the IEEE 16-bus system when closing branch 14.

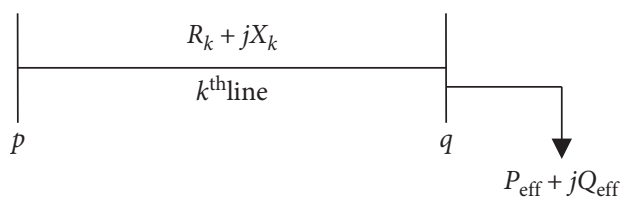

FIGURE 3: Simple distribution network line.

Now, the loss sensitivity factor (LSF) is obtained as follows:

$$
\frac{\delta P_{\text {lineloss }, k}}{\delta P_{\text {eff }}}=\frac{2 P_{\text {eff }}[q] \times R_{k}}{(V[q])^{2}} .
$$

From the result of power flow, LSF is calculated using (9) and arranged in the descending order. The nodes with high LSF are considered as the prior ones for installing DG units.

For the nodes in the list, their nominal voltage amplitude is configured by considering the fundamental voltage amplitude $($ norm $[i]=V[i] / 0.95)$. If the value norm $[i]$ of the $i$ th node is less than 1.01, that node is considered as a location for installing a DG unit. On the contrary, if the value norm [i] is greater than 1.01, it is not necessary to install any DG unit at that node, and that node will be also eliminated from a prior position from the list.

Depending on the requirements and scenarios considered in the problem, the LSF method or the SOS method can be applied to predetermine the locations for DG units.

\section{Symbiotic Organisms Search Algorithm}

The SOS algorithm is inspired by symbiotic relationships between two different organisms in the ecosystem. There are three common types of symbiotic relationships including mutualism, commensalism, and parasitism in nature. SOS mimics these symbiotic relationships to create new solutions. Similar to other population-based metaheuristic algorithms, SOS starts the search process with a population of organisms called the ecosystem, which is randomly generated. During the search process, each organism in the population continuously interacts with another one via three phases (mutualism, commensalism, and parasitism) to create new organisms. In each phase, the fitness value is calculated for each new organism. The update of a new organism is conducted only if its fitness value is evaluated better than its preinteraction fitness. After three phases, the best organism is selected for the next generation. The process of three phases is performed until the stopping criterion is met. Three phases of the SOS algorithm are now described.

5.1. Mutualism. Mutualism is a type of symbiotic relationship in which both organisms derive benefit in the interaction. An example of mutualism is the relationship between humans and plants. Humans inhale oxygen and exhale carbon dioxide, while plants absorb carbon dioxide and release oxygen. In this way, both plants and humans receive benefits from this relationship.

In SOS, a population of organisms (ecosystem) is represented by $X=\left[X_{1}, X_{2}, \ldots, X_{E c o_{-} s i z e}\right]^{T}$, in which each $\operatorname{organism} X_{i}=\left[x_{i 1}, x_{i 2}, \ldots, x_{i D}\right]\left(i=1,2, \ldots, E c o \_s i z e ; D\right.$ is the dimension of optimization problem; and Eco_size is the size of ecosystem). Let $X_{i}$ be an organism corresponding to the $i$ th member of the ecosystem. Another organism $X_{j}(j \neq i)$ is randomly selected from the ecosystem to interact with the organism $X_{i}$ through the mutualistic relationship. New 
organisms, $X_{i}^{\text {new }}$ and $X_{j}^{\text {new }}$, are created from this relationship as follows [27]:

$$
\begin{aligned}
X_{i}^{\text {new }} & =X_{i}+\operatorname{rand}(0,1) \times\left(X_{\text {best }}-M V \times b f_{1}\right), \\
X_{j}^{\text {new }} & =X_{j}+\operatorname{rand}(0,1) \times\left(X_{\text {best }}-M V \times b f_{2}\right), \\
M V & =\frac{b f_{1}+b f_{2}}{2},
\end{aligned}
$$

where rand $(0,1)$ is a random number in $[0,1] ; b f_{1}$ and $b f_{2}$ are the benefit factors (stochastically selected as either 1 or 2); $M V$ denotes a mutual vector which is the average of benefit factors, representing for a mutualistic relationship; and $X_{\text {best }}$ is the best organism in the ecosystem.

New organisms ( $X_{i}^{\text {new }}$ and $\left.X_{j}^{\text {new }}\right)$ are updated only if their fitness values are better than those of current organisms $\left(X_{i}\right.$ and $X_{j}$ ).

5.2. Commensalism. An example of commensalism is the interaction between remora and other fishes (such as sharks, mantas, and whales). The remoras can ride attached to other larger fishes because of a flat oval dish on their head. The remora detaches itself to eat the leftover of lager fishes. In this relationship, the remora gets benefits while the other larger fishes are unharmed. In the commensalism phase, organism $X_{j}$ is also randomly selected from the ecosystem to associate with organism $X_{i}$. From this interaction, organism $X_{i}$ tries to get benefit; however, organism $X_{j}$ neither benefits nor suffers from the relationship. A new organism, $X_{i}^{\text {new }}$, is produced based on commensal symbiosis as follows [27]:

$$
X_{i}^{\text {new }}=X_{i}+\operatorname{rand}(-1,1) \times\left(X_{\text {best }}-X_{j}\right) .
$$

A new organism, $X_{i}^{\text {new }}$, is updated if its fitness value of $X_{i}^{\text {new }}$ is better than the previous one.

5.3. Parasitism. The phase simulates a parasitic relationship where one organism is a parasite and gets benefit while the other is a host and gets harm. For instance, the Plasmodium parasite survives inside a human host through the bites of Anopheles mosquitoes. The parasite takes red blood cells of the human host to thrive and reproduce. As a result, the human host contracts malaria and possibly dies. In this relationship, the Plasmodium parasite receives benefit while the human gets harm.

In this phase, organism $X_{i}$ is assigned the role of a parasite. Organism $X_{j}$ is randomly chosen from the current ecosystem and serves as a host. Organism $X_{i}$ produces the Parasite_Vector $(P V)$ by duplicating itself. Some variables of $P V$ are randomly modified by random numbers to differentiate with organism $X_{i}$. After $P V$ is modified, fitness values are computed for both $P V$ and organisms $X_{j}$. If the fitness value of $P V$ is better than the fitness value of organisms $X_{j}$, it will replace organism $X_{j}$ in the current ecosystem. Otherwise, $P V$ will disappear, and organism $X_{j}$ is kept in that ecosystem [27].

\section{Implementation of SOS to the DNR Problem}

6.1. Initialization of Ecosystem. In the SOS algorithm, a population of organisms (ecosystem) is represented by $X=\left[X_{1}, X_{2}, \ldots, X_{E c o_{-} \text {size }}\right]^{T}$, in which each organism $X_{i}$ $\left(i=1,2, \ldots, E c o \_s i z e\right)$ represents a solution vector. The solution vector for the DNR problem considering the ODGP is expressed as follows:

$$
X_{i}=\left[S W_{1}^{i}, \ldots, S W_{N_{\mathrm{Tie}}}^{i}, x_{D G, 1}^{i}, \ldots, x_{D G, N_{D G}}^{i}, P_{D G, 1}^{i}, \ldots, P_{D G, N_{D G}}^{i}\right],
$$

where $S W_{1}^{i}, \ldots, S W_{N_{\text {Ti }}}^{i}$ are the tie-switches; $N_{\text {Tie }}$ is the number of tie-switches; $x_{D G, 1}^{i}, \ldots, x_{D G, N_{D G}}^{i}$ are the locations selected for the integration of DG units; and $P_{D G, 1}^{i}, \ldots, P_{D G, N_{D G}}^{i}$ are the size of DG units.

The organisms in the SOS method are randomly initialized within the search space. Thus, the solution variables for tie-switches $\left(S W_{i}\right)$, locations of DG units $\left(x_{D G, i}\right)$, and sizes of DG units $\left(P_{D G, i}\right)$ are generated as follows:

$$
\begin{array}{r}
S W_{i}=\operatorname{round}\left[S W_{\min , i}+\operatorname{rand}(0,1) \times\left(S W_{\max , i}-S W_{\min , i}\right)\right] ; \\
i=1, \ldots, N_{\mathrm{Tie}},
\end{array}
$$

$$
\begin{array}{r}
x_{D G, i}=\operatorname{round}\left[x_{D G \min , i}+\operatorname{rand}(0,1) \times\left(x_{D G \max , i}-x_{D G \min , i}\right)\right], \\
i=1, \ldots, N_{D G},
\end{array}
$$

$$
\begin{array}{r}
P_{D G, i}=P_{D G \min , i}+\operatorname{rand}(0,1) \times\left(P_{D G \max , i}-P_{D G \min , i}\right), \\
i=1, \ldots, N_{D G} .
\end{array}
$$

The tie-switches $\left(S W_{i}\right)$ and locations of DG units $\left(x_{D G, i}\right)$ are natural numbers. Therefore, they are rounded as seen in equation (15) and (16).

It is noted that, in equation (15), $S W_{\min }=1$ and $S W_{\max }$ is equal to the length of the $i$ th FL vectors. $S W_{i}$ is the ordinal number of one of switches in the ith FL. For instance, the first FL of the IEEE 16-bus system is $(1,3,7,9,10,11,14)$. The length of FL vector is 7 . Thus, $S W_{\max }=7$. After the optimization process, if $S W$ is found as 4, the solution of tie-switch is 9 .

6.2. Fitness Function. In the SOS method, each organism is evaluated by a fitness function which is computed by

$$
F_{T}=O F+K_{p} \sum_{i=1}^{N_{b}}\left(V_{i}-V_{i}^{\mathrm{lim}}\right)^{2}+K_{q} \sum_{i=1}^{N_{b r}}\left(I_{i}-I_{i}^{\lim }\right)^{2},
$$

where $K_{p}$ and $K_{q}$ are penalty factors for bus voltages and current capacity.

The limit values of the dependent variables (bus voltages and currents) can be presented as a general formula as follows:

$$
x^{\lim }= \begin{cases}x_{\max }, & \text { if } x>x_{\max }, \\ x_{\min }, & \text { if } x<x_{\min } \\ x, & \text { otherwise }\end{cases}
$$


where $x$ represents the values of $V_{i}$ and $I_{i}$ and $x^{\text {lim }}$ represents their limits, respectively.

6.3. Overall Procedure. The steps for implementation of the SOS algorithm for solving the DNR problem considering the ODGP are described as follows:

(i) Step 1: select the control parameters of SOS (D, Eco_size, maxiter).

(ii) Step 2: determine the fundamental loops for a distribution network, as in Section 3.1. Define upper and lower bounds.

(iii) Step 3: initialize randomly population of organisms $X$, as in Section 6.1.

(iv) Step 4: check the radial condition for each organism. Calculate the fitness function according to equation (18) for each organism. If the organism does not produce the radial configuration for the network, the fitness function is assigned to inf for that organism. Set Iter $=0$.

(v) Step 5: start the main loop, Iter $=$ Iter +1 .

(vi) Step 6: identify the best organism $X_{\text {best, }}$ which has the minimum fitness value.

(vii) Step 7: perform the mutualism phase, as in Section 5.1.

Randomly select an organism $X_{j}$ in the ecosystem $X$, where $X_{j} \neq X_{i}$.

Modify organisms $X_{i}$ and $X_{j}$ using equations (10)-(11) to form new organisms $X_{i}^{\text {new }}$ and $X_{j}^{\text {new }}$. The new organisms are checked for the radial condition and constraints, and a repairing strategy according to equation (19) is performed if any organism violates its bounds.

Calculate fitness value for each new organism using equation (18). Compare the fitness value of new organism with the old organism. Update the new organism in the ecosystem if its fitness value is better than the old one.

(viii) Step 8: perform the commensalism phase, as in Section 5.2.

Randomly select an organism $X_{j}$ in the ecosystem $X$, where $X_{j} \neq X_{i}$.

Modify organisms $X_{i}$ using (13) to form new organisms $X_{i}^{\text {new }}$.

The new organism is checked for the radial condition and constraints, and a repairing strategy according to equation (19) is performed if it violates its bounds.

Calculate the fitness value for the new organism using equation (18). Compare the fitness value of new organism with the old organism. Update the new organism in the ecosystem if its fitness value is better than the old one.

(ix) Step 9: perform the parasitism phase, as in Section 5.3.
Randomly select an organism $X_{j}$ in the ecosystem $X$, where $X_{j} \neq X_{i}$.

Create the Parasite_Vector $(P V)$ by duplicating the organism $X_{i}$.

The $P V$ is checked for the radial condition and constraints, and a repairing strategy according to equation (19) is performed if it violates its bounds.

Calculate fitness value for the $P V$ and organisms $X_{j}$ using equation (18). Compare the fitness value of the $P V$ with the organisms $X_{j}$. Replace organism $X_{j}$ with the $P V$ if the fitness value of $P V$ is better than the fitness value of organisms $X_{j}$.

(x) Step 10: go to Step 6 if Iter < maxIter. Otherwise, stop the algorithm.

In each phase (steps 7-9), the new organisms are checked for the radial condition and constraints, and a repairing strategy according to equation (19) is performed if any organism violates its bounds. The fitness function is calculated for each new organism using equation (18). The new organisms are updated by evaluating their fitness values.

\section{Numerical Results}

The effectiveness of the proposed SOS algorithm was evaluated and validated on 33-bus, 69-bus, 84-bus, and 118-bus RDNs. Matpower 5.1 toolbox was used to calculate the power flow in each test system. The proposed SOS algorithm was coded in MATLAB R2017b and run on an Intel Core i5 $2.5 \mathrm{GHz}$ CPU with $8 \mathrm{~GB}$ of RAM PC. In this study, SOS was run 30 independent trials to obtain the best solution. For each test system, seven different scenarios are considered for the DNR and ODGP problems as follows [23]:

(i) Scenario 1: considering the initial default network configuration (base case).

(ii) Scenario 2: only the DNR problem is performed.

(iii) Scenario 3: only the ODGP problem is performed

(iv) Scenario 4: the ODGP problem is performed after the DNR problem (using the result from Scenario 2).

(v) Scenario 5: the DNR problem is performed after the ODGP problem (using the result from Scenario 3).

(vi) Scenario 6: simultaneously considering the DNR problem and the optimal size of DG units. The locations of DG units are determined by the LFS method.

(vii) Scenario 7: simultaneously considering the DNR problem and the ODGP problem.

7.1. Setting Parameters of SOS. The SOS algorithm has only two controllable parameters including the population size (Eco_size) and the maximum number of iterations (maxIter). The setting of these parameters for the DNR problem is chosen by experiments. For each scenario, the Eco_size parameter is selected by varying from a low value and then 
gradually increased to collect different results. The number of Eco_size corresponding to the best result among different runs is considered as the proper value. In this study, the parameter Eco_size was varied from 50 to 250 with a step of 50. The parameter maxIter was chosen depending on the dimension and complexity of the problem. The optimal parameters of SOS for different scenarios of each test system were selected by experiments, as shown in Table 1 .

7.2.33-Bus Test Network. The first test system is the IEEE 33bus RDN [41], which is a small-scale power system including 37 branches, 32 sectionalizing switches, and 5 tie-line switches (33-34-35-36-37), as depicted in Figure 4. This system supplies power to the total load demand of $3.73 \mathrm{MW}$ and 2.3 MVAr. The nominal voltage of the system is $12.66 \mathrm{kV}$. Table 2 shows the result of the fundamental loops (FLs) for this system. Table 3 presents the results obtained from the proposed SOS and other methods such as ACSA [23], FWA [22], GWO-PSO [25], UVDA [42], and MPGSA [43] for seven scenarios.

As seen in Table 3, the real power loss from Scenario 1 (base case) was $202.68 \mathrm{~kW}$, which was reduced by SOS to $139.55 \mathrm{~kW}, 71.47 \mathrm{~kW}, 58.88 \mathrm{~kW}, 57.24 \mathrm{~kW}, 55.79 \mathrm{~kW}$, and $52.88 \mathrm{~kW}$ for Scenarios $2-7$, respectively. The corresponding percentages of power loss reduction (PLR) for these scenarios were $31.15 \%, 64.74 \%, 70.95 \%, 71.76 \%, 72.47 \%$, and $73.91 \%$, respectively. It can be seen that the percentage power loss reduction of Scenario 7 was the highest one, indicating that the real power loss of the system was significantly reduced when considering simultaneous the DNR and ODGP problems. On the contrary, the voltage profile of the system was improved from 0.913 p.u. (Scenario 1) to 0.971 p.u. (Scenario 7), as shown in Figure 5. Moreover, the voltage profile for all buses has been also depicted in Figure 5 for Scenario 2 to Scenario 7 where the Scenarios from 4 to 7 have provided better improvement than Scenarios 2 and 3. Therefore, Scenarios 4 to 7 are preferred in terms of the voltage improvement. Figure 6 shows the convergence curves yielded by the SOS method for Scenarios 2-7. Apparently, for Scenarios 2-6, the convergence curves converged around the 50th iteration. For Scenario 7, SOS reached the optimal solution at the 375 th iteration due to the complexity and large search space of the problem.

Also observed in Table 3, the results obtained by the SOS method were compared to those from ACSA [23], FWA [22], GWO-PSO [25], UVDA [42], and MPGSA [43] for all scenarios. For Scenario 2, the SOS algorithm found the optimal opened switches: 7-9-14-32-37, which offered the same power loss as the GWO-PSO method, approximate to MPGSA, and slightly lower than ACSA, FWA, and HSA methods. For Scenario 3, when three DG units were connected to the system with the opened switches: 33-34-35-3637 , the proposed SOS method found the optimal locations to install those DG units at buses 14,24 , and 30 with the optimal DG sizes of $0.7687 \mathrm{MW}, 1.0851 \mathrm{MW}$, and $1.0536 \mathrm{MW}$, resulting in the lowest real power loss among compared methods. For Scenario 4, after the network reconfiguration, DG units were installed at buses 8,24 , and
30 with the corresponding optimal DG sizes of $0.9356 \mathrm{MW}$, $1.0665 \mathrm{MW}$, and $0.9527 \mathrm{MW}$. SOS obtained a better real power loss than other methods and close to the result from ACSA for this scenario. For Scenario 5, with the optimal placement of DG units in Scenario 3, the SOS method optimized the network configuration with the opened switches: 7-8-9-32-37. For this scenario, SOS offered the lowest power loss as compared to the ACSA and FWA methods. For Scenario 6, the DG units were installed at buses 8,13 , and 25 . The optimal DG sizes obtained by SOS were $0.6776 \mathrm{MW}, 0.6502 \mathrm{MW}$, and $1.6293 \mathrm{MW}$. The opened switches: 6-10-27-32-34 was also found by SOS to form the optimal network configuration. Among compared methods, SOS obtained the best power loss for this scenario. For Scenario 7, when both DNR and ODGP problems were simultaneously considered, SOS found the optimal network configuration with the opened switches: 6-11-28-31-34. DG units were integrated to the optimal locations at buses 8,18 , and 25 with the optimal sizes of $0.7932 \mathrm{MW}, 0.6690 \mathrm{MW}$, and $1.4029 \mathrm{MW}$, respectively. The real power loss obtained by SOS was slightly lower than that from ACSA for this scenario. It can be observed from Table 3 that the proposed SOS method obtained a very good result quality for the IEEE 33-bus system for all scenarios.

The obtained result for Scenario 7 of this system by the proposed SOS including the location and size of DGs and the location of opened switches is represented in Figure 7. In this figure, the three DGs are located at buses 8,18 , and 25 with the corresponding capacity of $0.7932 \mathrm{MW}, 0.669 \mathrm{MW}$, and $1.4029 \mathrm{MW}$, respectively while the opened switches are at locations of $6,11,28,31$, and 34 .

7.3. 69-Bus Test Network. The IEEE 69-bus RDN is the second test system that consists of 73 branches, 68 sectionalizing switches, and 5 tie-line switches (69-70-71-72-73) [44]. The active and reactive power load demands of this network are $3.802 \mathrm{MW}$ and 2.69 MVAr, respectively. This system operates at a voltage level of $12.66 \mathrm{kV}$. Figure 8 describes the single line diagram of the IEEE 69-bus RDN, and Table 4 shows FLs for this system.

Table 5 shows the results obtained by the SOS method and other methods including tie-switches, locations and sizes of DG units, power loss, loss reduction percentage, and minimum voltage for the IEEE 69-bus RDN with different scenarios. Accordingly, Scenario 1 (base case) had a power loss of $225.03 \mathrm{~kW}$, which was reduced by SOS to $98.62 \mathrm{~kW}$, $69.44 \mathrm{~kW}, 35.17 \mathrm{~kW}, 39.17 \mathrm{~kW}, 42.55 \mathrm{~kW}$, and $35.20 \mathrm{~kW}$ for Scenarios $2-7$, respectively. The corresponding percentages of power losses reduction were $56.17 \%, 69.14 \%, 84.37 \%$, $82.59 \%, 79.17 \%$, and $84.35 \%$. As observed in Table 6, the voltage magnitude was increased from 0.9092 p.u. (Scenario 1) to 0.9810 p.u. (Scenario 7). Figures 9 and 10 show the voltage magnitude at all buses and the convergence curves for all scenarios, respectively. Similar to the case for the 33bus test system, the voltage improvement provided by the proposed method is shown in Figure 9 for the 7 different scenarios. As observed from the figure, the voltage improvement from Scenarios 2 and 3 are worse than that from 
TABLE 1: The parameters of SOS for each test system.

\begin{tabular}{|c|c|c|c|c|c|c|c|c|}
\hline \multirow{2}{*}{ System } & \multicolumn{2}{|c|}{ 33-bus } & \multicolumn{2}{|c|}{ 69-bus } & \multicolumn{2}{|c|}{ 84-bus } & \multicolumn{2}{|c|}{ 118-bus } \\
\hline & Eco_size & maxIter & Eco_size & maxIter & Eco_size & maxIter & Eco_size & maxiter \\
\hline Scenario 2 & 50 & 500 & 50 & 500 & 250 & 1000 & 100 & 1250 \\
\hline Scenario 3 & 50 & 500 & 50 & 500 & 100 & 1000 & 100 & 1000 \\
\hline Scenario 4 & 100 & 1000 & 100 & 500 & 100 & 1000 & 100 & 1000 \\
\hline Scenario 5 & 50 & 500 & 50 & 500 & 50 & 1000 & 50 & 1000 \\
\hline Scenario 6 & 100 & 500 & 50 & 500 & 100 & 1000 & 100 & 1000 \\
\hline Scenario 7 & 100 & 500 & 50 & 2000 & 100 & 1000 & 100 & 1000 \\
\hline
\end{tabular}

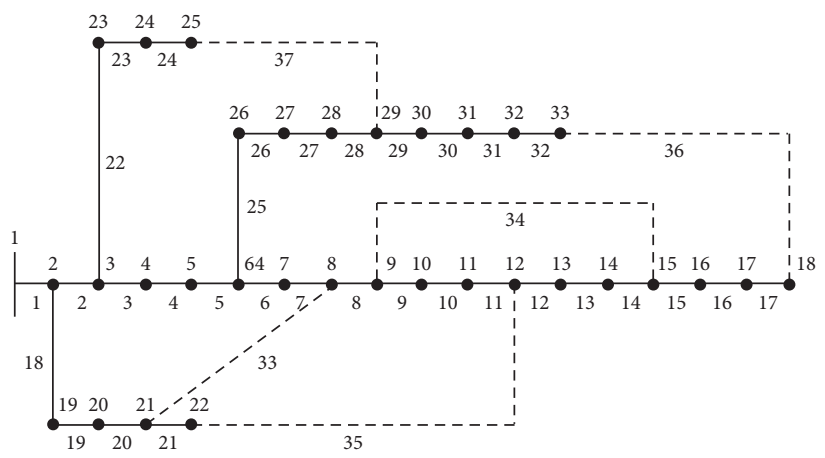

Figure 4: The IEEE 33-bus system.

TABLE 2: Fundamental loops for the IEEE 33-bus system.

\begin{tabular}{lcc}
\hline FL & Switches & Length of FL \\
\hline 1 & $2,3,4,5,6,7,18,19,20,33$ & 10 \\
2 & $9,10,11,12,13,14,34$ & 7 \\
3 & $2,3,4,5,6,7,8,9,10,11,18,19,20,21,35$ & 15 \\
4 & $6,7,8,9,10,11,12,13,14,15,16,17,25,26,27,28,29,30,31,32,36$ & 21 \\
5 & $3,4,5,22,23,24,25,26,27,28,37$ & 11 \\
\hline
\end{tabular}

other scenarios. Therefore, Scenarios 4 to 7 are preferred in terms of the voltage improvement.

The results obtained by the SOS method were compared to those from ACSA [23], FWA [22], GWO-PSO [25], and UVDA [42], as shown in Table 5. For Scenario 2, without any DG units, SOS optimized the network configuration with the opened switches: 14-55-61-69-70. The real power loss obtained after the network reconfiguration was $98.62 \mathrm{~kW}$, which was close to the results from other methods. For Scenario 3, without network reconfiguration, SOS determined three buses 11,18 , and 61 for the installation of DG units. The corresponding optimal DG sizes were $0.4832 \mathrm{MW}$, $0.3916 \mathrm{MW}$, and 1.7282 MW. SOS provided the lowest real power loss as compared to other methods for this scenario. For Scenario 4, with the opened switches of the network as in Scenario 2, SOS found the optimal DG sizes of $0.5085 \mathrm{MW}$, $1.4282 \mathrm{MW}$, and 0.5024 MW for the DG installation at buses 11,61 , and 64. After the DG installation, the real power loss of the system was $35.17 \mathrm{~kW}$. SOS achieved the best result for this scenario as compared to other methods. For Scenario 5, after DG units were installed as in Scenario 3, SOS determined the opened switches: 12-56-64-69-70 for the DNR problem, resulting in a real power loss of $39.17 \mathrm{~kW}$, which was close to FWA, and better than ACSA. For Scenario 6,
DG units were connected to buses 59, 60, and 61. SOS found the optimal DG sizes of $0.3939 \mathrm{MW}, 0.4121 \mathrm{MW}$, and 1.098 MW. The opened switches 69-70-12-55-26 were also determined by SOS for the optimal network configuration. For Scenario 7, SOS provided the optimal network configuration with the opened switches: 14-56-61-69-70 and obtained the optimal locations at buses 11, 61, and 65 with the corresponding optimal sizes of $0.5398 \mathrm{MW}, 1.4428 \mathrm{MW}$, and 0.5304 for DG placement. The power loss by SOS was lower than ACSA for this scenario. This indicated that the proposed SOS method is an effective method for solving the complex problem of both DNR and ODGP.

The obtained result for Scenario 7 of this system by the proposed SOS including the location and size of DGs and the location of opened switches is represented in Figure 11. In this figure, the three DGs are located at buses 11, 61, and 65 with the corresponding capacity of $0.5398 \mathrm{MW}, 1.4428 \mathrm{MW}$, and $0.5304 \mathrm{MW}$, respectively, while the opened switches are at locations of $16,56,61,69$, and 70 .

7.4. 84-Bus Test Network. The 84-bus distribution network consists of 96 branches, 83 sectionalizing switches, and 13 tie-line switches with a nominal voltage of $11.4 \mathrm{kV}$, as shown 
TABle 3: Comparative results for the IEEE 33-bus system with seven scenarios.

\begin{tabular}{|c|c|c|c|c|c|c|c|}
\hline Scenario & Item & ACSA [23] & FWA [22] & GWO-PSO [25] & UVDA [42] & MPGSA [43] & SOS \\
\hline \multirow{3}{*}{1} & Opened switches & - & - & - & - & - & $33-34-35-36-37$ \\
\hline & $P_{L}(\mathrm{~kW})$ & - & - & - & - & - & 202.68 \\
\hline & $V_{\min }$ (p.u.) & - & - & - & - & - & 0.913 \\
\hline \multirow{5}{*}{2} & Opened switches & $7-14-9-32-28$ & $7-14-9-32-28$ & $7-9-14-32-37$ & $7-9-14-32-37$ & $7-9-14-32-37$ & $7-9-14-32-37$ \\
\hline & $P_{L}(\mathrm{~kW})$ & 139.98 & 139.98 & 138.93 & 139.55 & 139.5 & 139.55 \\
\hline & PLR (\%) & 30.93 & 30.93 & 33.35 & 31.15 & 31.16 & 31.15 \\
\hline & $V_{\min }$ (p.u.) & 0.9413 & 0.9413 & 0.9423 & 0.9378 & 0.9343 & 0.9378 \\
\hline & Time $(\mathrm{s})$ & - & - & 30.61 & - & - & 165.2 \\
\hline \multirow{7}{*}{3} & Opened switches & $33-34-35-36-37$ & $33-34-35-36-37$ & $33-34-35-36-37$ & $33-34-35-36-37$ & $33-34-35-36-37$ & $33-34-35-36-37$ \\
\hline & & $0.7798 /(14)$ & $0.5897 /(14)$ & $0.1070 /(18)$ & $0.875 /(11)$ & $0.1058 /(17)$ & $0.7687 /(14)$ \\
\hline & $P_{D G}(\mathrm{MW}) /($ node $)$ & $1.1251 /(24)$ & $0.1895 /(18)$ & $0.5724 /(17)$ & $0.925 /(29)$ & $0.5900 /(18)$ & $1.0851 /(24)$ \\
\hline & & $1.3496 /(30)$ & $1.0146 /(32)$ & $1.0462 /(33)$ & $0.931 /(24)$ & $1.0812 /(33)$ & $1.0536 /(30)$ \\
\hline & $P_{L}(\mathrm{~kW})$ & 74.26 & 88.68 & 96.76 & 74.213 & 95.42 & 71.47 \\
\hline & PLR (\%) & 63.26 & 56.24 & 52.26 & 63.39 & 52.92 & 64.74 \\
\hline & $V_{\min }$ (p.u.) & 0.9778 & 0.9680 & 0.9670 & 0.962 & 0.9585 & 0.9687 \\
\hline \multirow{7}{*}{4} & Opened switches & $7-14-9-32-28$ & $7-14-9-32-28$ & $7-9-14-32-37$ & $7-9-14-32-37$ & $7-9-14-32-37$ & $7-9-14-32-37$ \\
\hline & & $1.7536 /(29)$ & $0.5996 /(32)$ & $0.2686 /(32)$ & $1.125 /(30)$ & $0.2469 /(31)$ & $0.9356 /(8)$ \\
\hline & $P_{D G}(\mathrm{MW}) /($ node $)$ & $0.5397 /(12)$ & $0.3141 /(33)$ & $0.1611 /(31)$ & $0.592 /(15)$ & $0.1795 /(32)$ & $0.9527 /(30)$ \\
\hline & & $0.5045 /(16)$ & $0.1591 /(18)$ & $0.6612 /(30)$ & $0.526 /(12)$ & $0.6645 /(33)$ & $1.0665 /(24)$ \\
\hline & $P_{L}(\mathrm{~kW})$ & 58.79 & 83.91 & 97.13 & 66.602 & 92.87 & 58.88 \\
\hline & PLR (\%) & 71.00 & 58.59 & 52.07 & 67.14 & 54.17 & 70.95 \\
\hline & $V_{\min }$ (p.u.) & 0.9802 & 0.9612 & 0.9479 & 0.9758 & 0.9482 & 0.9741 \\
\hline \multirow{7}{*}{5} & Opened switches & $33-9-8-36-27$ & $7-34-9-32-28$ & - & - & - & $7-8-9-32-37$ \\
\hline & & $0.7798 /(14)$ & $0.5897 /(14)$ & - & - & - & $0.7687 /(14)$ \\
\hline & $P_{D G}(\mathrm{MW}) /($ node $)$ & $1.1251 /(24)$ & $0.1895 /(18)$ & - & - & - & $1.0851 /(24)$ \\
\hline & & $1.3496 /(30)$ & $1.0146 /(32)$ & - & - & - & $1.0536 /(30)$ \\
\hline & $P_{L}(\mathrm{~kW})$ & 62.98 & 68.28 & - & - & - & 57.24 \\
\hline & PLR (\%) & 68.93 & 66.31 & - & - & - & 71.76 \\
\hline & $V_{\min }$ (p.u.) & 0.9826 & 0.9712 & 一 & - & - & 0.9710 \\
\hline \multirow{7}{*}{6} & Opened switches & $7-10-13-32-27$ & $7-14-11-32-28$ & $7-14-10-32-28$ & $7-10-13-27-32$ & $7-14-10-28-31$ & $6-10-27-32-34$ \\
\hline & & $0.4263 /(32)$ & $0.5367 /(32)$ & $0.5258 /(32)$ & $1.554 /(29)$ & $0.6311 /(18)$ & $1.6293 /(25)$ \\
\hline & $P_{D G}(\mathrm{MW}) /($ node $)$ & $1.2024 /(29)$ & $0.6158 /(29)$ & $0.5586 /(31)$ & $0.649 /(15)$ & $0.5568 /(32)$ & $0.6776 /(8)$ \\
\hline & & $0.7127 /(18)$ & $0.5315 /(18)$ & $0.5840 /(33)$ & $0.486 /(21)$ & $0.5986 /(33)$ & $0.6502 /(13)$ \\
\hline & $P_{L}(\mathrm{~kW})$ & 63.69 & 67.11 & 73.05 & 57.287 & 72.23 & 55.79 \\
\hline & $\operatorname{PLR}(\%)$ & 68.58 & 66.89 & 63.95 & 71.74 & 64.36 & 72.47 \\
\hline & $V_{\min }$ (p.u.) & 0.9786 & 0.9713 & 0.9700 & 0.9676 & 0.9724 & 0.9721 \\
\hline \multirow{7}{*}{7} & Opened switches & $33-34-11-31-28$ & - & - & - & - & $6-11-28-31-34$ \\
\hline & & $0.8968 /(18)$ & - & - & - & - & $0.7932 /(8)$ \\
\hline & $P_{D G}(\mathrm{MW}) /($ node $)$ & $1.4381 /(25)$ & - & - & - & - & $0.669 /(18)$ \\
\hline & & $0.9646 /(7)$ & - & - & - & - & $1.4029 /(25)$ \\
\hline & $P_{L}(\mathrm{~kW})$ & 53.21 & - & - & - & - & 52.88 \\
\hline & PLR (\%) & 73.75 & - & - & - & - & 73.91 \\
\hline & $V_{\min }$ (p.u.) & 0.9806 & - & - & - & - & 0.9710 \\
\hline
\end{tabular}

in Figure 12 [45]. The total power load demand of the system is $28.35 \mathrm{MW}$ and 20.70 MVAr. The FLs are determined for the system, as given in Table 6 . The initial opened switches of this system are 84-85-86-87-88-89-90-91-92-93-94-95-96.

Table 7 presents the results obtained by SOS for different scenarios. As seen from this table, the real power loss of the initial network configuration was $531.99 \mathrm{~kW}$. This power loss was reduced to $469.88 \mathrm{~kW}, 359.85 \mathrm{~kW}, 345.42 \mathrm{~kW}$, $334.74 \mathrm{~kW}, 341.10 \mathrm{~kW}$, and $327.81 \mathrm{~kW}$ by the proposed SOS, respectively. For Scenario 2, SOS found the optimal structure of the network with the opened switches: 55-7-86-72-13-8990-83-92-39-34-42-62. For Scenario 3, DG units were installed at bus 7 with a size of $3.0595 \mathrm{MW}$, bus 72 with a size of $2.8539 \mathrm{MW}$, and bus 80 with a size of $3.6018 \mathrm{MW}$. For
Scenario 4, with the network configuration in Scenario 2, SOS determined the optimal DG locations (buses 20, 54, and 80) with the optimal sizes of $3.4924 \mathrm{MW}, 3.0103 \mathrm{MW}$, and 3.1347 MW, respectively. For Scenario 5, with the allocation of DG units in Scenario 3, SOS found the opened switched: 34-39-42-55-85-86-87-88-89-90-91-92-96 for the optimal network reconfiguration. For Scenario 6, the DG locations were determined as buses 21,54 , and 71 . SOS found the optimal DG sizes of $3.3178 \mathrm{MW}, 3.1536 \mathrm{MW}$, and 2.8591 MW to install those DG units. Simultaneously, SOS also optimized the network configuration with the opened switches: 7-14-34-39-42-61-82-84-86-87-88-90-92. For Scenario 7, when the DNR problem is simultaneously considered with the ODGP problem, SOS determined the 


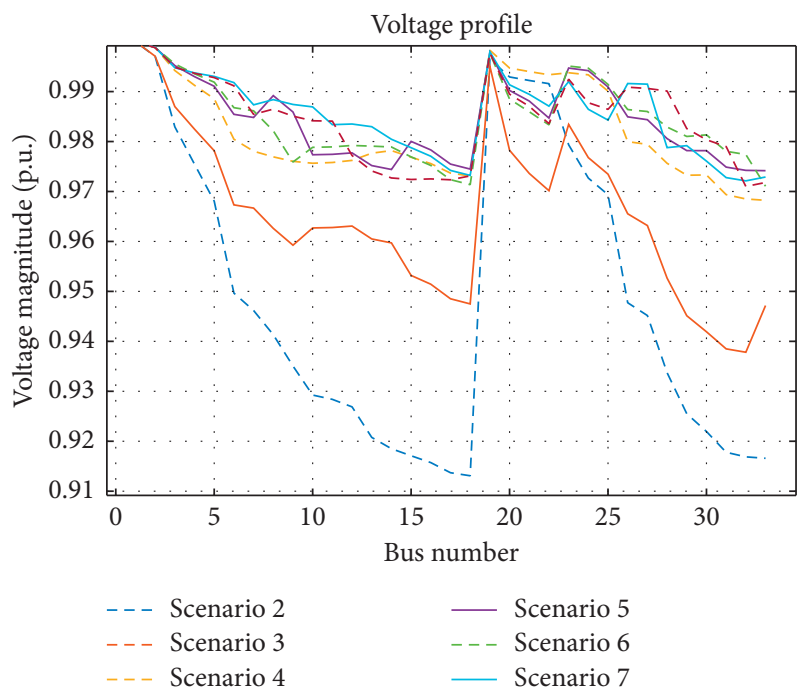

FIgURE 5: Voltage profile improvements of Scenarios 2-7 by SOS for the IEEE 33-bus system.

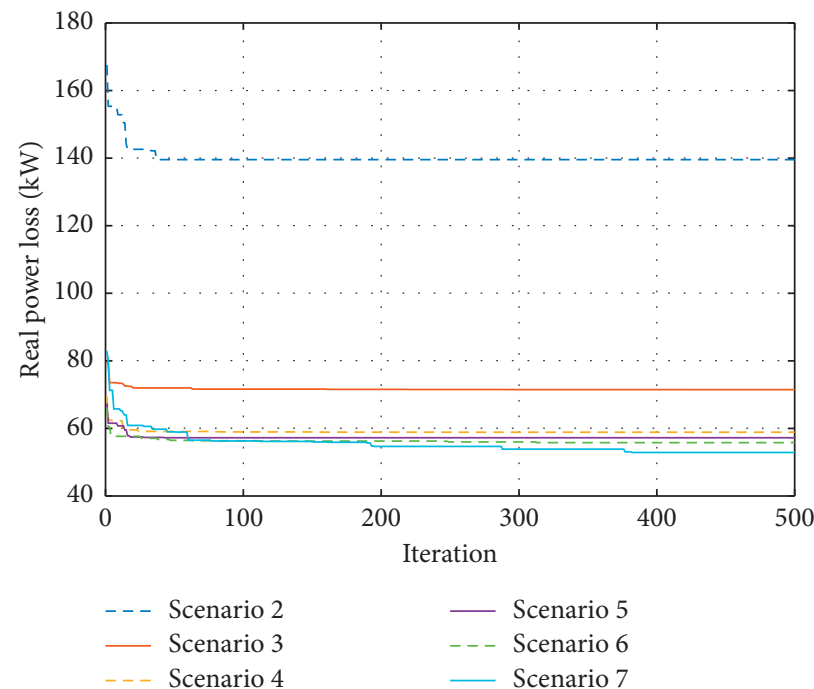

Figure 6: Convergence curves of Scenarios 2-7 by SOS for the IEEE 33-bus system.

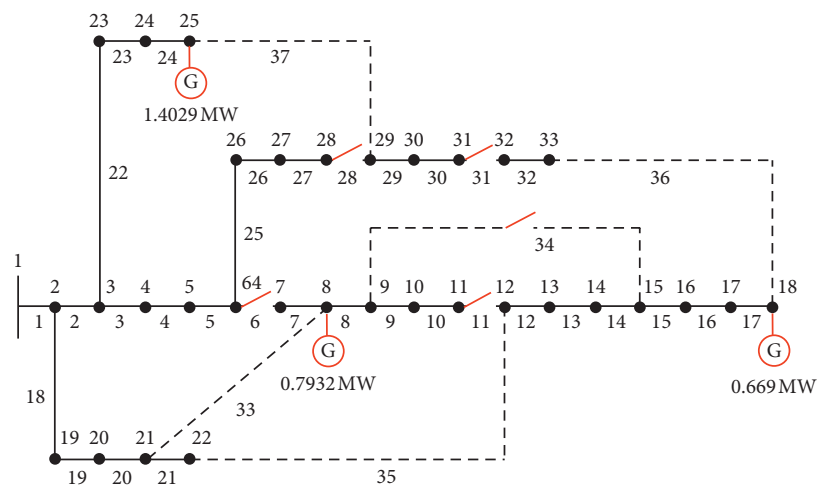

Figure 7: The result representation of Scenario 7 for the IEEE 33-bus system. 


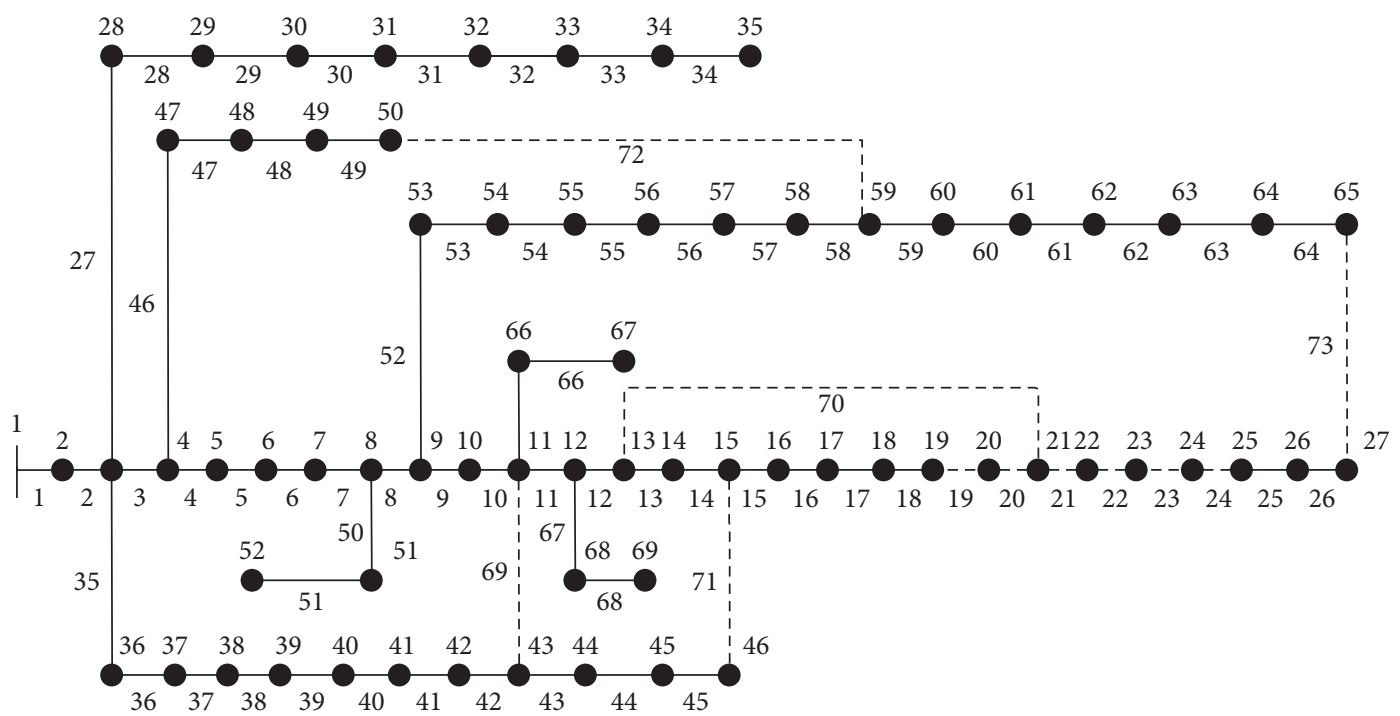

Figure 8: The IEEE 69-bus system.

TABLE 4: Fundamental loops for the IEEE 69-bus system.

\begin{tabular}{lcc}
\hline FL & Switches & Length of FL \\
\hline 1 & $3,4,5,6,7,8,9,10,35,36,37,38,39,40,41,42,69$ & 17 \\
2 & $13,14,15,16,17,18,19,20,70$ & 9 \\
3 & $3,4,5,6,7,8,9,10,11,12,13,14,35,36,37,38,39,40,41,42,43,44,45,71$ & 24 \\
4 & $4,5,6,7,8,46,47,48,49,52,53,54,55,56,57,58,72$ & 17 \\
5 & $9,10,11,12,13,14,15,16,17,18,19,20,21,22,23,24,25,26,52,53,54,55,56,57,58,59,60,61,62,63,64,73$ & 32 \\
\hline
\end{tabular}

TABLE 5: Comparative results for the IEEE 69-bus system with seven cases.

\begin{tabular}{|c|c|c|c|c|c|c|}
\hline Scenario & Item & ACSA [23] & FWA [22] & GWO-PSO [25] & UVDA [42] & SOS \\
\hline \multirow{3}{*}{1} & Opened switches & - & - & - & - & $69-70-71-72-73$ \\
\hline & $P_{L}(\mathrm{~kW})$ & - & - & - & - & 225.03 \\
\hline & $V_{\min }$ (p.u.) & - & 一 & - & - & 0.9092 \\
\hline \multirow{4}{*}{2} & Opened switches & $69-70-14-57-61$ & 69-70-14-56-61 & $69-18-13-56-61$ & $14-58-61-69-70$ & $14-55-61-69-70$ \\
\hline & $P_{L}(\mathrm{~kW})$ & 98.59 & 98.56 & 99.35 & 98.58 & 98.62 \\
\hline & PLR (\%) & 56.16 & 56.16 & 55.85 & 56.19 & 56.17 \\
\hline & $V_{\min }$ (p.u.) & 0.9495 & 0.9495 & 0.9428 & 0.9495 & 0.9495 \\
\hline \multirow{7}{*}{3} & Opened switches & $69-70-71-72-73$ & $69-70-71-72-73$ & $69-70-71-72-73$ & $69-70-71-72-73$ & $69-70-71-72-73$ \\
\hline & & $0.6022 /(11)$ & $0.4085 /(65)$ & $0.1018 /(65)$ & $1.410 /(61)$ & $0.4832 /(11)$ \\
\hline & $P_{D G}(\mathrm{MW}) /($ node $)$ & $0.3804 /(18)$ & $1.1986 /(61)$ & $0.3690 /(64)$ & $0.604 /(11)$ & $1.7282 /(61)$ \\
\hline & & $2.0000 /(61)$ & $0.2258 /(27)$ & $1.3024 /(63)$ & $0.417 /(17)$ & $0.3916 /(18)$ \\
\hline & $P_{L}(\mathrm{~kW})$ & 72.44 & 77.85 & 86.77 & 72.626 & 69.44 \\
\hline & PLR (\%) & 67.79 & 65.39 & 61.43 & 67.72 & 69.14 \\
\hline & $V_{\min }$ (p.u.) & 0.9890 & 0.9740 & 0.9677 & 0.9688 & 0.9791 \\
\hline \multirow{7}{*}{4} & Opened switches & $69-70-14-57-61$ & 69-70-14-56-61 & $69-18-13-56-61$ & $14-58-61-69-70$ & $14-56-61-69-70$ \\
\hline & & $1.7254 /(61)$ & $1.0014 /(61)$ & $1.0666 /(61)$ & $1.378 /(61)$ & $1.4282 /(61)$ \\
\hline & $P_{D G}(\mathrm{MW}) /($ node $)$ & $0.4666 /(64)$ & $0.2145 /(62)$ & $0.3525 /(60)$ & $0.62 /(11)$ & $0.5085 /(11)$ \\
\hline & & $0.3686 /(12)$ & $0.1425 /(64)$ & $0.4257 /(58)$ & $0.722 /(64)$ & $0.5024 /(64)$ \\
\hline & $P_{L}(\mathrm{~kW})$ & 37.23 & 43.88 & 51.3 & 37.84 & 35.17 \\
\hline & PLR (\%) & 83.45 & 80.49 & 77.2 & 83.18 & 84.37 \\
\hline & $V_{\min }$ (p.u.) & 0.9870 & 0.9720 & 0.9619 & 0.9801 & 0.9812 \\
\hline
\end{tabular}


TABle 5: Continued.

\begin{tabular}{|c|c|c|c|c|c|c|}
\hline Scenario & Item & ACSA [23] & FWA [22] & GWO-PSO [25] & UVDA [42] & SOS \\
\hline \multirow{7}{*}{5} & Opened switches & $69-70-12-58-61$ & $69-70-12-58-61$ & - & - & $12-56-64-69-70$ \\
\hline & & $0.6022 /(11)$ & $0.4085 /(65)$ & - & - & $0.4832 /(11)$ \\
\hline & $P_{D G}(\mathrm{MW}) /($ node $)$ & $0.3804 /(18)$ & $1.1986 /(61)$ & - & - & $1.7282 /(61)$ \\
\hline & & $2.0000 /(61)$ & $0.2258 /(27)$ & - & - & $0.3916 /(18)$ \\
\hline & $P_{L}(\mathrm{~kW})$ & 41.13 & 39.69 & - & - & 39.17 \\
\hline & $P L R(\%)$ & 81.71 & 82.35 & - & - & 82.59 \\
\hline & $V_{\min }$ (p.u.) & 0.9828 & 0.9763 & - & - & 0.9771 \\
\hline \multirow{7}{*}{6} & Opened switches & $69-70-12-58-61$ & $69-70-13-55-63$ & $69-17-13-58-61$ & $14-58-63-69-70$ & $12-56-64-69-70$ \\
\hline & & $1.7496 /(61)$ & $1.1272 /(61)$ & $1.0666 /(61)$ & $1.472 /(61)$ & $0.3939 /(59)$ \\
\hline & $P_{D G}(\mathrm{MW}) /($ node $)$ & $0.1566 /(62)$ & $0.2750 /(62)$ & $0.3525 /(60)$ & $0.538 /(11)$ & $1.098 /(61)$ \\
\hline & & $0.4090 /(65)$ & $0.4159 /(65)$ & $0.4257 /(62)$ & $0.673 /(17)$ & $0.4121 /(60)$ \\
\hline & $P_{L}(\mathrm{~kW})$ & 40.49 & 39.25 & 40.3 & 37.11 & 42.55 \\
\hline & PLR (\%) & 82.0 & 82.55 & 82.08 & 83.51 & 79.17 \\
\hline & $V_{\min }$ (p.u.) & 0.9873 & 0.9796 & 0.9736 & 0.9816 & 0.9772 \\
\hline \multirow{7}{*}{7} & Opened switches & $69-70-14-58-61$ & - & - & - & $14-56-61-69-70$ \\
\hline & & $0.5413 /(11)$ & - & - & - & $1.4428 /(61)$ \\
\hline & $P_{D G}(\mathrm{MW}) /($ node $)$ & $0.5536 /(65)$ & - & - & - & $0.5398 /(11)$ \\
\hline & & $1.7240 /(61)$ & - & - & - & $0.5304 /(65)$ \\
\hline & $P_{L}(\mathrm{~kW})$ & 37.02 & - & - & - & 35.20 \\
\hline & PLR (\%) & 83.54 & - & - & - & 84.35 \\
\hline & $V_{\min }$ (p.u.) & 0.9869 & - & 一 & - & 0.9815 \\
\hline
\end{tabular}

TABLE 6: Fundamental loops for the 84-bus system.

\begin{tabular}{lcc}
\hline FL & Switches & Length of FL \\
\hline 1 & $1,2,3,4,5,47,48,49,50,51,52,53,54,55,84$ & 15 \\
2 & $1,2,3,4,5,6,7,56,57,58,59,60,85$ & 13 \\
3 & $11,43,86$ & 3 \\
4 & $11,12,65,66,67,68,69,70,71,72,87$ \\
5 & $11,12,13,73,74,75,76,88$ \\
6 & $11,12,14,15,16,17,18,89$ & 8 \\
7 & $15,16,25,26,90$ & 8 \\
8 & $15,16,17,18,19,20,77,78,79,80,81,82,83,91$ & 5 \\
9 & $25,26,27,28,30,31,32,92$ & 14 \\
\hline
\end{tabular}

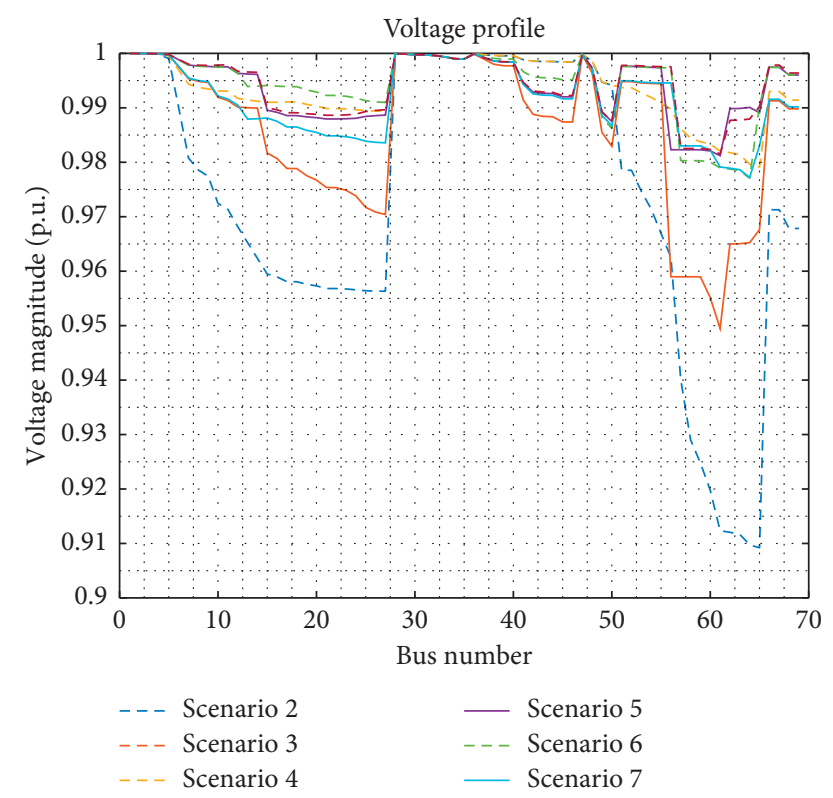

FIGURE 9: Voltage profile improvements of Scenarios 2-7 by SOS for the IEEE 69-bus system. 


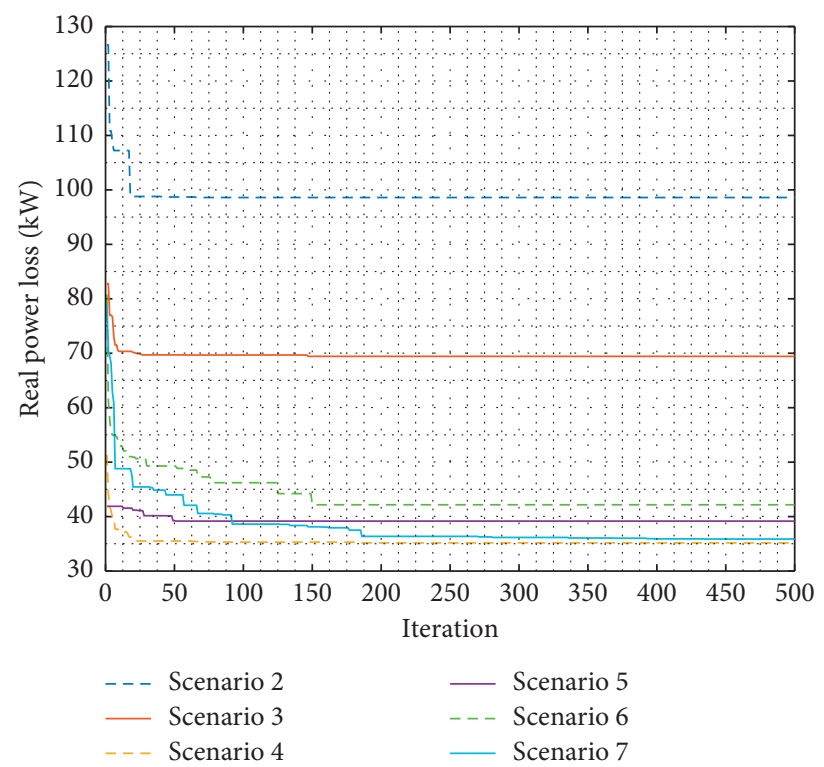

FIgURE 10: Convergence curves of Scenarios 2-7 by SOS for the IEEE 69-bus system.

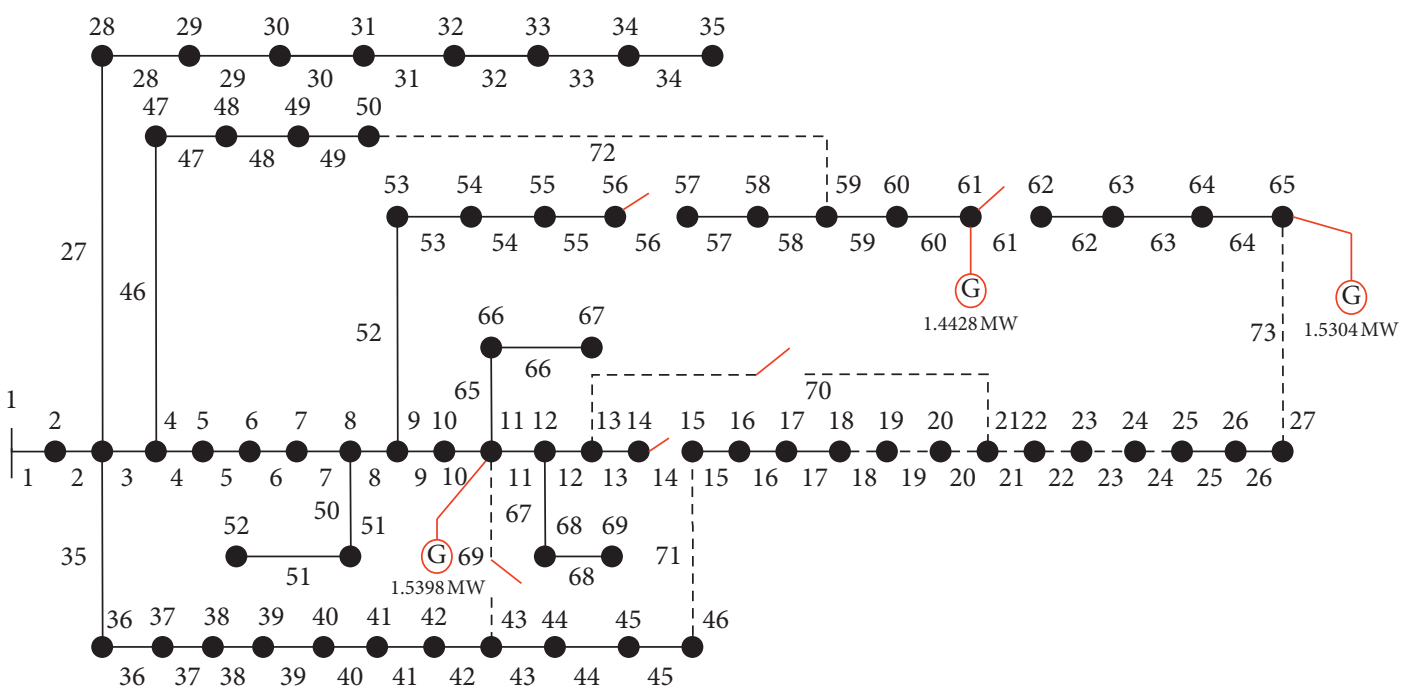

FIgURE 11: The result representation of Scenario 7 for the IEEE 69-bus system.

optimal opened switches: 14-34-39-42-63-72-81-84-85-8688-90-92 for the DNR. Simultaneously, SOS found the optimal DG locations at buses 7, 20, and 54, corresponding with the optimal sizes of $3.1644 \mathrm{MW}, 4.736 \mathrm{MW}$, and $2.9421 \mathrm{MW}$ for the DG installation. The percentages of power loss reduction correspond to $11.68 \%, .36 \%, 35.07 \%$, $37.08 \%, 35.88 \%$, and $38.38 \%$ for Scenarios $2-7$, respectively. The voltage profile was improved by SOS from 0.9285 p.u. (Scenario 1) to 0.9532 p.u., 0.9557 p.u., 0.9532 p.u., 0.9527 p.u., 0.9561 p.u., and 0.9532 p.u. for Scenarios 2-7, respectively. Among these scenarios, Scenario 7 offered the highest power loss, which implied that the simultaneous consideration of DNR and ODGP problems offered better power loss reduction than other cases. Figure 13 shows the voltage profiles for all scenarios, and Figure 14 depicts the convergence curves obtained by SOS for Scenarios 2-7. In
Figure 13, the voltage improvement from Scenario 2 is worse than the others while the other scenarios have provided the similar voltage improvement.

For Case 2, the results obtained by SOS for this system were compared with those from AIS-ACO [46], Ordinal optimization (OO) [16], PGSA [47], MIHDE [45], and GA [48], as shown in Table 8. The proposed SOS method found the optimal network configuration with the opened switches: 55-7-86-72-13-89-90-83-92-39-34-42-62, resulting in the real power loss of $469.88 \mathrm{~kW}$, which was lower than the real power loss obtained by AIS-ACO. The SOS method provided a power loss equal to that from GA, PGSA, and MIHDE.

7.5.118-Bus Test Network. The IEEE 118-bus RDN is a largescale system, operating at $11 \mathrm{kV}$. This system consists of 132 


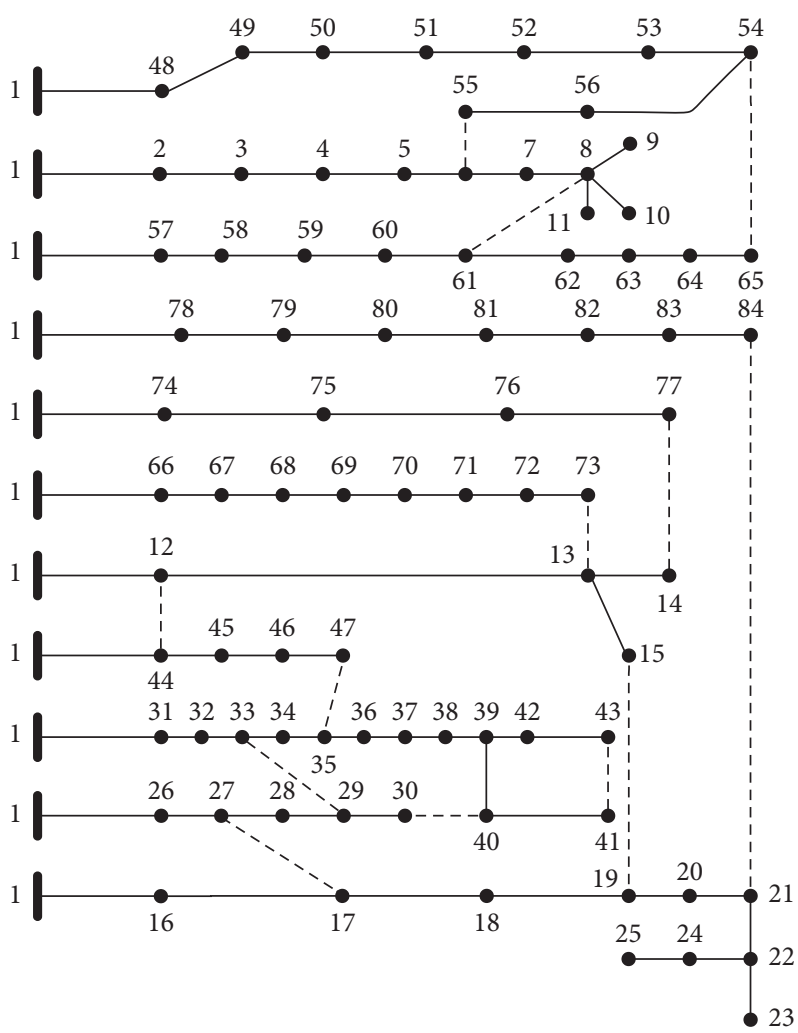

Figure 12: The 84-bus system.

TABLE 7: Results of the 84-bus system for seven cases.

\begin{tabular}{|c|c|c|}
\hline Scenario & Item & SOS \\
\hline \multirow{3}{*}{1} & Opened switches & $84-85-86-87-88-89-90-91-92-93-94-95-96$ \\
\hline & $P_{L}(\mathrm{~kW})$ & 531.99 \\
\hline & $V_{\min }$ (p.u.) & 0.9285 \\
\hline \multirow{4}{*}{2} & Opened switches & $55-7-86-72-13-89-90-83-92-39-34-42-62$ \\
\hline & $P_{L}(\mathrm{~kW})$ & 469.88 \\
\hline & PLR (\%) & 11.68 \\
\hline & $V_{\min }$ (p.u.) & 0.9532 \\
\hline \multirow{5}{*}{3} & Opened switches & $84-85-86-87-88-89-90-91-92-93-94-95-96$ \\
\hline & $P_{D G}(\mathrm{MW}) /($ node $)$ & $3.6018 /(80) ; 3.0595 /(7) ; 2.8539 /(72)$ \\
\hline & $P_{L}(\mathrm{~kW})$ & 359.85 \\
\hline & PLR (\%) & 32.36 \\
\hline & $V_{\min }$ (p.u.) & 0.9557 \\
\hline \multirow{5}{*}{4} & Opened switches & $55-7-86-72-13-89-90-83-92-39-34-42-62$ \\
\hline & $P_{D G}(\mathrm{MW}) /($ node $)$ & $3.1347 /(80) ; 3.0103 /(54) ; 3.4924 /(20)$ \\
\hline & $P_{L}(\mathrm{~kW})$ & 345.42 \\
\hline & PLR (\%) & 35.07 \\
\hline & $V_{\min }$ (p.u.) & 0.9532 \\
\hline \multirow{5}{*}{5} & Opened switches & $34-39-42-55-85-86-87-88-89-90-91-92-96$ \\
\hline & $P_{D G}(\mathrm{MW}) /($ node $)$ & $3.6018 /(80) ; 3.0595 /(7) ; 2.8539 /(72)$ \\
\hline & $P_{L}(\mathrm{~kW})$ & 334.74 \\
\hline & PLR (\%) & 37.08 \\
\hline & $V_{\min }$ (p.u.) & 0.9561 \\
\hline \multirow{5}{*}{6} & Opened switches & $7-14-34-39-42-61-82-84-86-87-88-90-92$ \\
\hline & $P_{D G}(\mathrm{MW}) /($ node $)$ & $3.3178 /(21) ; 2.8591 /(71) ; 3.1536 /(54)$ \\
\hline & $P_{L}(\mathrm{~kW})$ & 341.10 \\
\hline & PLR (\%) & 35.88 \\
\hline & $V_{\min }$ (p.u.) & 0.9561 \\
\hline \multirow{5}{*}{7} & Opened switches & $14-34-39-42-63-72-81-84-85-86-88-90-92$ \\
\hline & $P_{D G}(\mathrm{MW}) /($ node $)$ & $3.1644 /(7) ; 2.9421 /(54) ; 4.736 /(20)$ \\
\hline & $P_{L}(\mathrm{~kW})$ & 327.81 \\
\hline & PLR (\%) & 38.38 \\
\hline & $V_{\min }$ (p.u.) & 0.9532 \\
\hline
\end{tabular}




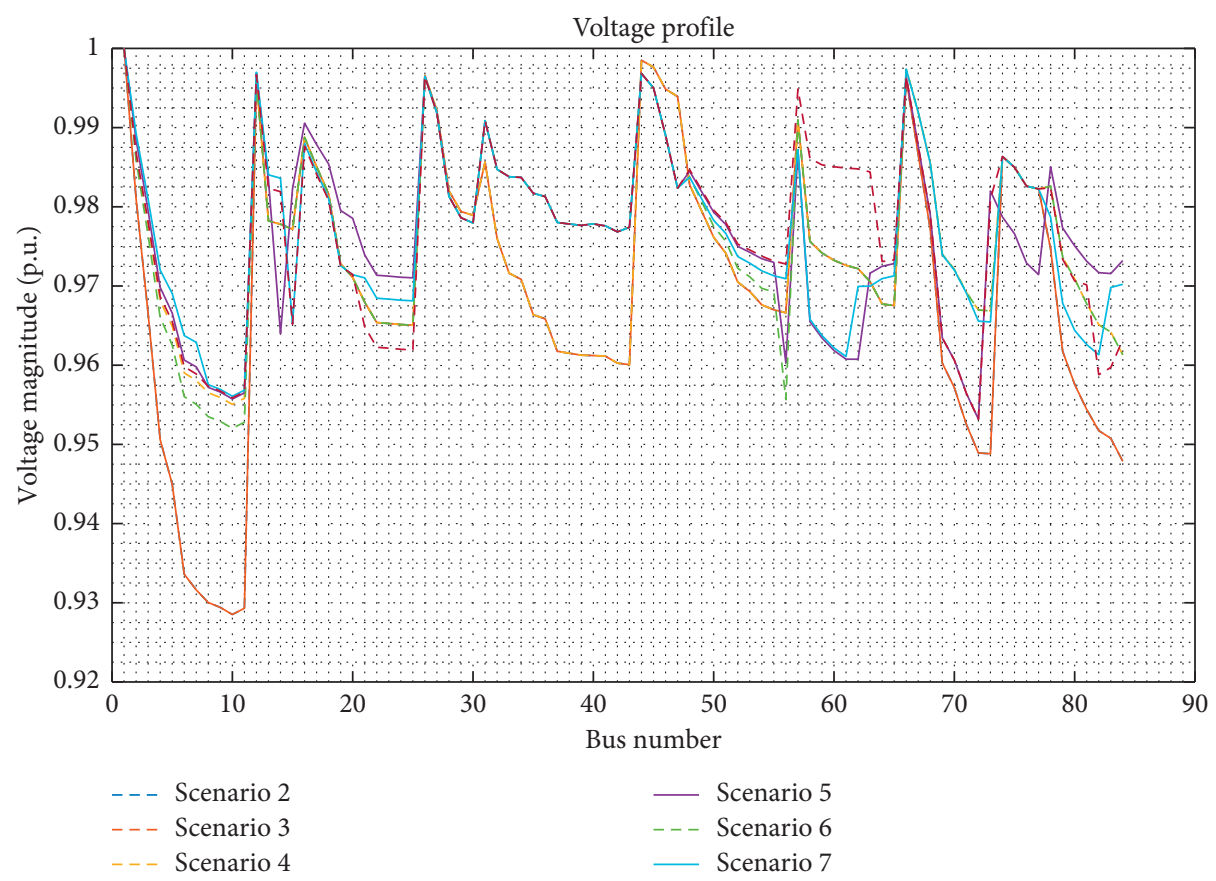

FIGURE 13: Voltage profile improvements of Scenarios 2-7 by SOS for the 84-bus system.

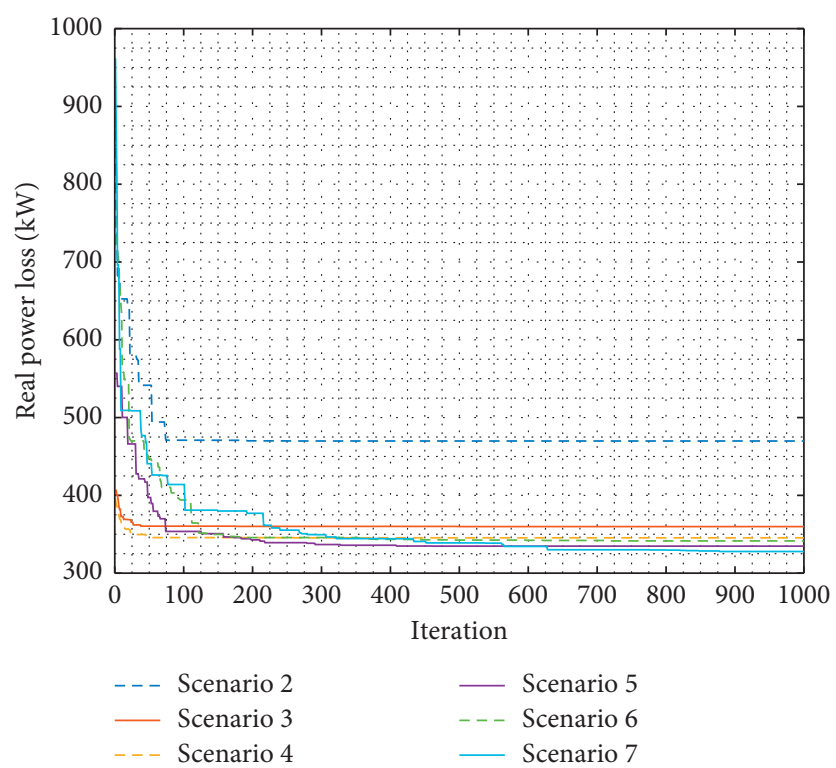

Figure 14: Convergence curves of Scenarios $2-7$ by SOS for the 84-bus system.

TABLE 8: Result comparison of the 84-bus network for Case 2.

\begin{tabular}{lccc}
\hline Method & Opened switches & Power loss $(\mathrm{kW})$ & $V_{\min }(\mathrm{p} . \mathrm{u})$ \\
\hline Initial & $84-85-86-87-88-89-90-91-92-93-94-95-96$ & 531.99 & 0.9285 \\
AIS-ACO [46] & $7-13-34-39-42-55-62-72-86-89-90-91-92$ & 471.14 & 0.9479 \\
OO [16] & $7-33-38-42-63-72-83-84-86-88-89-90-92$ & 471.79 & - \\
PGSA [47] & $55-7-86-72-13-89-90-83-92-39-34-42-62$ & 469.88 & - \\
MIHDE [45] & $55-7-86-72-13-89-90-83-92-39-34-42-62$ & 469.88 & - \\
GA [48] & $55-7-86-72-13-89-90-83-92-39-34-42-62$ & 469.88 & 36.15 \\
SOS & $55-7-86-72-13-89-90-83-92-39-34-42-62$ & 469.88 & - \\
\hline
\end{tabular}




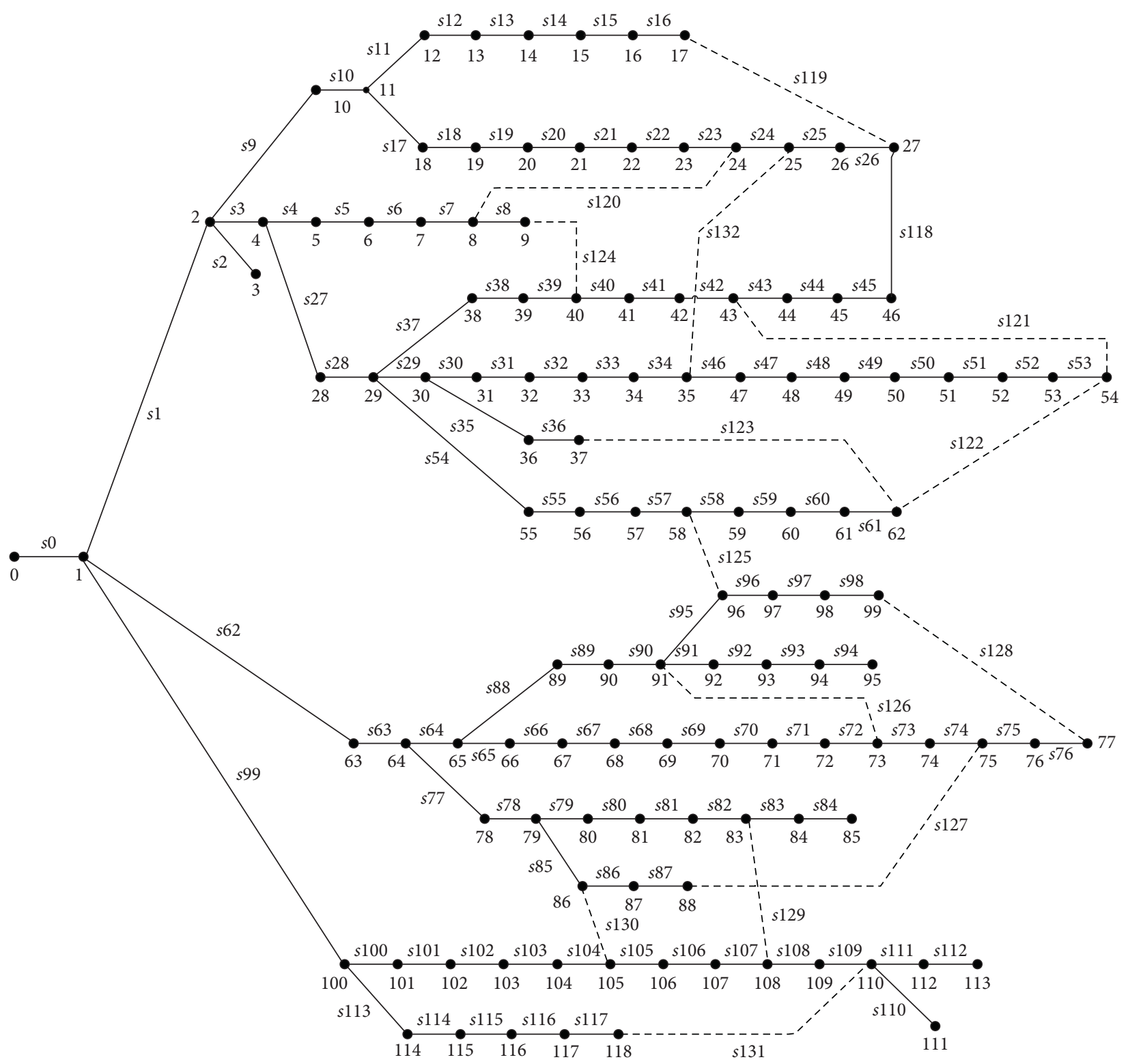

Figure 15: The single line diagram of the IEEE 118-bus system.

branches, 118 sectionalizing switches, and 15 tie-line switches [14]. The total power load demand of the system is 22,709 MW and 17,041 MVAr. Figure 15 describes the single line diagram for this system, and Table 9 shows the FLs of this system.

Table 10 presents the results obtained by the SOS method for different scenarios. For Scenario 1, the system had a real power loss of $1298.09 \mathrm{~kW}$ with a minimum voltage of 0.8688 p.u. For Scenarios $2-7$, the real power loss was reduced to $854.04 \mathrm{~kW}, 667.54 \mathrm{~kW}, 617.38 \mathrm{~kW}, 611.96 \mathrm{~kW}$, $624.15 \mathrm{~kW}$, and $571.47 \mathrm{~kW}$, respectively. For Scenario 2, SOS determined the optimal opened switches: 42-25-23-121-5058-39-95-71-74-97-129-130-109-34 for the optimal structure of the network. For Scenario 3, DG units were connected to the network at buses 50,71, and 110 with the optimal sizes of $2.8769 \mathrm{MW}, 3.0287 \mathrm{MW}$, and 2.8991 MW. For Scenario 4, with the network configuration in Scenario 2,
SOS found the optimal locations (buses 29,79, and 110) to install DG units. The optimal DG sizes were 9.404 MW, 3.1954 MW, and 2.2942 MW, respectively. For Scenario 5, with the optimal placement of DG units in Scenario 3, SOS optimized the network reconfiguration with the opened switched: 21-25-33-39-42-43-53-60-70-74-76-85-107-125129. For Scenario 6, the locations to install DG units were buses 78,89 , and 118 . SOS determined the optimal DG sizes of $3.4754 \mathrm{MW}, 2.8687 \mathrm{MW}$, and 2.2824 MW. Simultaneously, SOS also optimized the network structure with the opened switches: 23-25-34-39-43-50-61-70-73-76-109-121125-129-130. For Scenario 7, when both DNR and ODGP problems were simultaneously considered, SOS found the optimal opened switches: 23-25-34-39-42-43-50-61-70-8285-86-97-108-125. The optimal DG locations were buses 29, 73 , and 110, along with the optimal DG sizes of 8.5973 MW, 3.5043 MW, and 2.4212 MW, which were found by SOS for 
TABLe 9: Fundamental loops for the 118-bus system.

\begin{tabular}{lcc}
\hline FL & Switches & Length of FL \\
\hline 1 & $3,9,10,17,18,19,20,21,22,23,24,25,26,27,28,37,38,39,40,41,42,43,44,45,118$ & 25 \\
2 & $11,12,13,14,15,16,17,18,19,20,21,22,23,24,25,26,119$ & 17 \\
3 & $3,4,5,6,7,9,10,17,18,19,20,21,22,23,120$ & 15 \\
4 & $29,30,31,32,33,34,37,38,39,40,41,42,46,47,48,49,50,51,52,53,121$ & 23 \\
5 & $29,30,31,32,33,34,46,47,48,49,50,51,52,53,54,55,56,57,58,59,60,61,122$ & 12 \\
6 & $29,35,36,54,55,56,57,58,59,60,61,123$ & 11 \\
7 & $4,5,6,7,8,27,28,37,38,39,124$ \\
8 & $1,3,27,28,54,55,56,57,62,63,64,88,89,90,95,125$ & 12 \\
9 & $65,66,67,68,69,70,71,72,88,89,90,126$ & 17 \\
10 & $64,65,66,67,68,69,70,71,72,73,74,77,78,85,86,87,127$ & 20 \\
11 & $65,66,67,68,69,70,71,72,73,74,75,76,88,89,90,95,96,97,98,128$ \\
12 & $62,63,77,78,79,80,81,82,99,100,101,102,103,104,105,106,107,129$ \\
13 & $62,63,77,78,85,99,100,101,102,103,104,130$ & 18 \\
14 & $100,101,102,103,104,105,106,107,108,109,113,114,115,116,117,131$ & 12 \\
15 & $3,9,10,17,18,19,20,21,22,23,24,27,28,29,30,31,32,33,34,132$ \\
\hline
\end{tabular}

TABle 10: Results of the IEEE 118-bus system for seven cases.

\begin{tabular}{|c|c|c|}
\hline Scenario & Item & SOS \\
\hline \multirow{3}{*}{1} & Opened switches & $118-119-120-121-122-123-124-125-126-127-128-129-130-131-132$ \\
\hline & $P_{L}(\mathrm{~kW})$ & 1298.09 \\
\hline & $V_{\min }$ (p.u.) & 0.8688 \\
\hline \multirow{4}{*}{2} & Opened switches & $42-25-23-121-50-58-39-95-71-74-97-129-130-109-34$ \\
\hline & $P_{L}(\mathrm{~kW})$ & 854.04 \\
\hline & PLR (\%) & 34.21 \\
\hline & $V_{\min }$ (p.u.) & 0.9323 \\
\hline \multirow{5}{*}{3} & Opened switches & $118-119-120-121-122-123-124-125-126-127-128-129-130-131-132$ \\
\hline & $P_{D G}(\mathrm{MW}) /($ node $)$ & $2.8769 /(50) ; 2.8991 /(110) ; 3.0287 /(71)$ \\
\hline & $P_{L}(\mathrm{~kW})$ & 667.54 \\
\hline & PLR (\%) & 48.58 \\
\hline & $V_{\min }$ (p.u.) & 0.9541 \\
\hline \multirow{5}{*}{4} & Opened switches & $42-25-23-121-50-58-39-95-71-74-97-129-130-109-34$ \\
\hline & $P_{D G}(\mathrm{MW}) /($ node $)$ & $3.1954 /(79) ; 2.2942 /(110) ; 9.404 /(29)$ \\
\hline & $P_{L}(\mathrm{~kW})$ & 617.38 \\
\hline & $\operatorname{PLR}(\%)$ & 52.44 \\
\hline & $V_{\min }$ (p.u.) & 0.9444 \\
\hline \multirow{5}{*}{5} & Opened switches & $21-25-33-39-42-43-53-60-70-74-76-85-107-125-129$ \\
\hline & $P_{D G}(\mathrm{MW}) /($ node $)$ & $2.8769 /(50) ; 2.8991 /(110) ; 3.0287 /(71)$ \\
\hline & $P_{L}(\mathrm{~kW})$ & 611.96 \\
\hline & PLR (\%) & 52.86 \\
\hline & $V_{\min }$ (p.u.) & 0.9611 \\
\hline \multirow{5}{*}{6} & Opened switches & $23-25-34-39-43-50-61-70-73-76-109-121-125-129-130$ \\
\hline & $P_{D G}(\mathrm{MW}) /($ node $)$ & $3.4754 /(78) ; 2.8687 /(89) ; 2.2824 /(118)$ \\
\hline & $P_{L}(\mathrm{~kW})$ & 624.15 \\
\hline & $\operatorname{PLR}(\%)$ & 51.92 \\
\hline & $V_{\min }$ (p.u.) & 0.9533 \\
\hline \multirow{5}{*}{7} & Opened switches & $23-25-34-39-42-43-50-61-70-82-85-86-97-108-125$ \\
\hline & $P_{D G}(\mathrm{MW}) /($ node $)$ & $8.5973 /(29) ; 3.5043 /(73) ; 2.4212 /(110)$ \\
\hline & $P_{L}(\mathrm{~kW})$ & 571.47 \\
\hline & $\operatorname{PLR}(\%)$ & 55.98 \\
\hline & $V_{\min }$ (p.u.) & 0.9594 \\
\hline
\end{tabular}

the DG installation. As also seen in Table 10, the percentages of power loss reduction were $34.21 \%, 48.58 \%, 52.44 \%$, $52.86 \%, 51.92 \%$, and $55.98 \%$ for Scenarios $2-7$, respectively, showing the highest power loss reduction for Scenario 7. This indicated that the simultaneous consideration of both DNR and ODGP significantly reduced the real power loss of the system. The minimum values of voltage magnitude were 0.9323 p.u., 0.9540 p.u., 0.9444 p.u., 0.9611 p.u., 0.9533 p.u., and 0.9594 p.u. for Scenarios 2-7, respectively. Figure 16 shows the voltage profile improvement of the system for all scenarios, and Figure 17 depicts the convergence characteristics of the proposed SOS method for Scenarios 2-7. As 


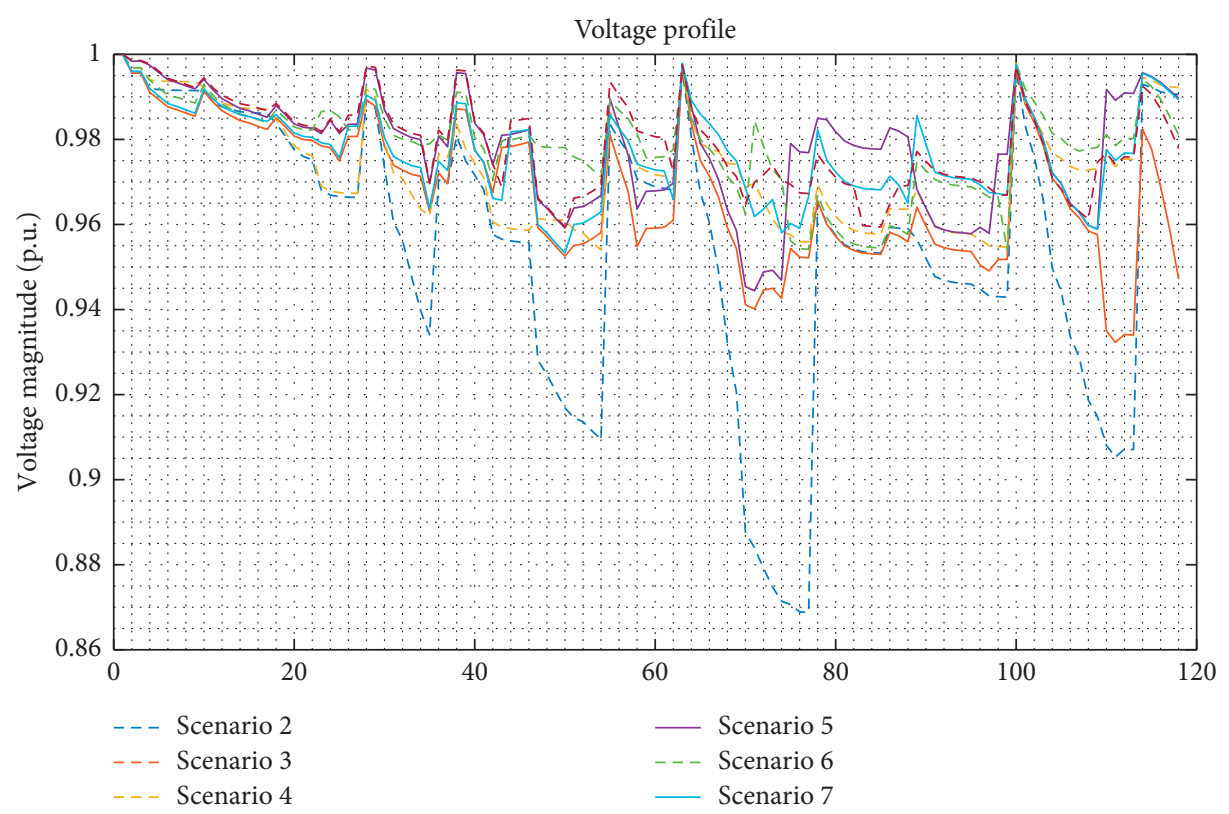

FIgURE 16: Voltage profile improvements of Scenarios 2-7 by SOS for the IEEE 118-bus system.

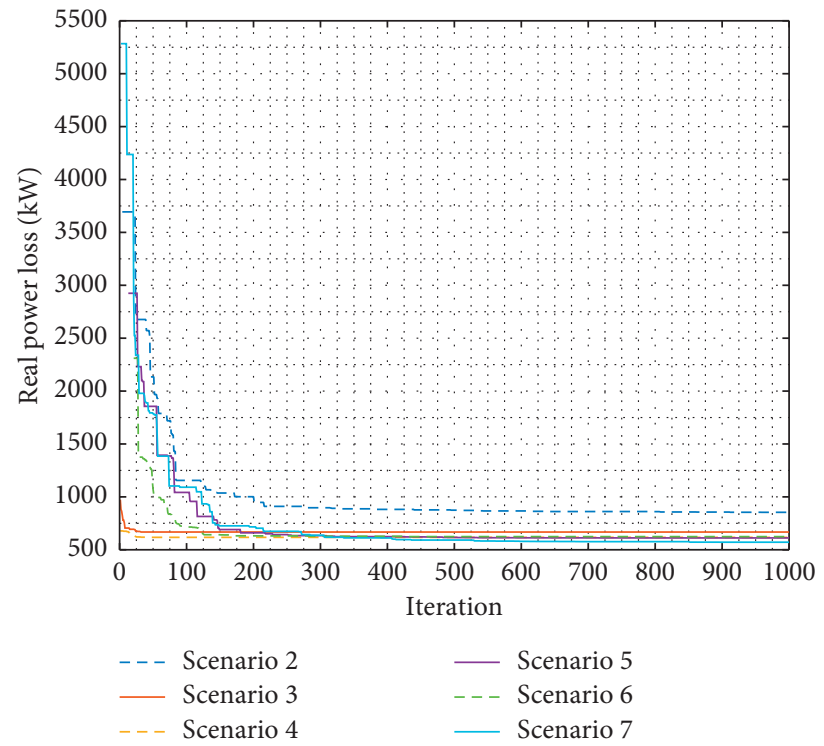

Figure 17: Convergence curves of Scenarios 2-7 by SOS for the 118-bus system.

observed from Figure 16, the voltage improvement from Scenarios 4 to 7 is better than that from Scenarios 2 and 3, where Scenario 2 is the worst case. Therefore, Scenario 2 is worse than others for all test systems for voltage improvement at buses.

For Case 2, Table 11 shows the results obtained by the SOS algorithm and other methods including ITS [14], MTS [40], FWA [22], CSA [17], HSA [15], and FA [18]. As seen in this Table, SOS obtained a power loss of $854.04 \mathrm{~kW}$, which was less than the results from ITS and MTS, and close to FWA, HSA, CSA, and FA. It indicated that the SOS method is very effective for dealing with the large-scale DNR problem.
7.6. Comparison of Computational Time. The SOS algorithm was executed on a $3.5 \mathrm{GHz} 8$-core PC with $16 \mathrm{~GB}$ of RAM. Table 12 shows the computational time of SOS for each scenario of each test system. The computation time of SOS was compared with the computational of GWO-PSO [25] for Scenario 2 of the 33-bus test network, and OO [16], MIHDE [45], and GA [48] for Scenario 2 of the 84-bus test network. It can be seen that SOS required longer computation time than GWO-PSO, OO, MIHDE, and GA. However, SOS is able to find better results than the compared methods. The fact is that SOS also offered better quality solution than most compared optimization methods for different test system. 
TABLE 11: Result comparison of the IEEE 119-bus network for Case 2.

\begin{tabular}{lccc}
\hline Methods & Opened switches & Power loss $(\mathrm{kW})$ & $V_{\text {min }}(\mathrm{p} . \mathrm{u})$ \\
\hline Initial & $118-119-120-121-122-123-124-125-126-127-128-129-130-131-132$ & 1298.09 \\
ITS [14] & $44-26-24-53-51-62-42-100-78-75-102-113-110-115-36$ & 865.865 \\
MTS [40] & $44-26-24-53-51-62-42-100-78-75-102-113-110-115-36$ & 865.865 \\
FWA [22] & $42-25-23-121-50-58-39-95-71-74-97-129-130-109-34$ & 854.06 \\
CSA [17] & $42-25-23-121-50-58-39-95-71-74-97-129-130-109-34$ & 855.04 & 0.9323 \\
HSA [15] & $23-27-33-43-53-62-72-75-123-125-126-129-130-131-132$ & 854.21 & 0.9323 \\
FA [18] & $24-26-35-40-43-51-59-72-75-96-98-110-122-130-131$ & 854.06 & 0.9298 \\
SOS & $42-25-23-121-50-58-39-95-71-74-97-129-130-109-34$ & 854.04 & 0.9323 \\
\hline
\end{tabular}

TABLE 12: Computational time of SOS for each test system.

\begin{tabular}{lcccc}
\hline System & 33-bus & 69-bus & 84 -bus & 118 -bus \\
\hline Scenario 2 & 165.2 & 344.7 & 741.8 & 364.3 \\
Scenario 3 & 595.6 & 872.6 & 4106.4 & 5608.1 \\
Scenario 4 & 591.3 & 1783.1 & 4168.2 & 5606.8 \\
Scenario 5 & 124.2 & 205.5 & 501.6 & 195.6 \\
Scenario 6 & 988.5 & 1153.4 & 5444.2 & 2151.3 \\
Scenario 7 & 741.2 & 1970.4 & 2856.2 & 1512.2 \\
\hline
\end{tabular}

It is noted that the computational time may not be directly compared among the methods due to different computer processors and programming languages used. Therefore, the key factor for the result comparison is mainly the objective function value rather than the computational time.

\section{Conclusion}

In this paper, an approach based on the SOS algorithm and the graph theory has been presented to reconfigure and integrate DG units simultaneously in distribution networks with the aim of minimizing real power loss. The graph theory helped the SOS algorithm reduce infeasible network configurations and checked the radial constraint of each new generated configuration during the optimization process. This strategy significantly reduces the computational time of the SOS in solving the DNR problem. The proposed method was tested on different distribution networks including 33bus, 69-bus, 84-bus, and 119-bus. Different scenarios of the DNR and ODGP problems were considered to evaluate the effectiveness of the proposed method. It was found that Scenario VII (considering simultaneously the DNR and ODGP problems) offered the best real power loss reduction compared to other scenarios. For this scenario, the power loss reductions in 33-bus, 69-bus, 84-bus, and 119-bus networks are $73.91 \%, 84.35 \%, 38.38 \%$, and $55.98 \%$, respectively. Moreover, from the result comparisons, it was shown that the proposed SOS algorithm provided better solution quality than many other optimization methods, especially for large-scale systems. Consequently, the SOS algorithm becomes an effective approach for dealing with the network reconfiguration and DG placement problems in distribution networks.

This study offers a decision tool based on the SOS algorithm for distribution network operators (DNOs) in the planning of distribution networks. The DNOs can estimate the reconfiguration network pattern and the optimal locations and sizes of DG units to effectively operate distribution networks. An effective planning helps the DNOs save operational costs and defer or avoid network reinforcements.

\section{Data Availability}

No data were used to support this study.

\section{Conflicts of Interest}

The authors declare that there are no conflicts of interest regarding the publication of this paper.

\section{Acknowledgments}

This research was funded by Ho Chi Minh City University of Technology (HCMUT), VNU-HCM, under grant number BK-SDH-2020-8141223.

\section{References}

[1] S. Gopiya Naik, D. K. Khatod, and M. P. Sharma, "Optimal allocation of combined DG and capacitor for real power loss minimization in distribution networks," International Journal of Electrical Power \& Energy Systems, vol. 53, pp. 967-973, 2013.

[2] J. Zhu, X. Xiong, J. Zhang, G. Shen, Q. Xu, and Y. Xue, “A rule based comprehensive approach for reconfiguration of electrical distribution network," Electric Power Systems Research, vol. 79, no. 2, pp. 311-315, 2009.

[3] A. Merlin and H. Back, "Search for a minimal-loss operating spanning tree configuration in an urban power distribution system," in Proceedings of the 5th Power System Computation Conference (PSCC), Porto, Portugal, June 1975.

[4] S. Civanlar, J. J. Grainger, H. Yin, and S. S. H. Lee, "Distribution feeder reconfiguration for loss reduction," IEEE Transactions on Power Delivery, vol. 3, no. 3, pp. 1217-1223, 1988.

[5] J. A. Martín and A. J. Gil, "A new heuristic approach for distribution systems loss reduction," Electric Power Systems Research, vol. 78, no. 11, pp. 1953-1958, 2008.

[6] V. N. Gohokar, M. K. Khedkar, and G. M. Dhole, "Formulation of distribution reconfiguration problem using network topology: a generalized approach," Electric Power Systems Research, vol. 69, no. 2-3, pp. 304-310, 2004.

[7] J. Z. Zhu, "Optimal reconfiguration of electrical distribution network using the refined genetic algorithm," Electric Power Systems Research, vol. 62, no. 1, pp. 37-42, 2002. 
[8] J. Mendoza, R. Lopez, D. Morales, E. Lopez, P. Dessante, and R. Moraga, "Minimal loss reconfiguration using genetic algorithms with restricted population and addressed operators: real application," IEEE Transactions on Power Systems, vol. 21, no. 2, pp. 948-954, 2006.

[9] J. Torres, J. L. Guardado, F. Rivas-Dávalos, S. Maximov, and E. Melgoza, "A genetic algorithm based on the edge window decoder technique to optimize power distribution systems reconfiguration," International Journal of Electrical Power \& Energy Systems, vol. 45, no. 1, pp. 28-34, 2013.

[10] W.-C. Wu and M.-S. Tsai, "Application of enhanced integer coded Particle Swarm optimization for distribution system feeder reconfiguration," IEEE Transactions on Power Systems, vol. 26, no. 3, pp. 1591-1599, 2011.

[11] N. Gupta, A. Swarnkar, and K. R. Niazi, "Reconfiguration of distribution systems for real power loss minimization using adaptive particle swarm optimization," Electric Power Components and Systems, vol. 39, no. 4, pp. 317-330, 2011.

[12] L. Li and C. Xuefeng, "Distribution network reconfiguration based on niche binary Particle Swarm optimization algorithm," Energy Procedia, vol. 17, pp. 178-182, 2012.

[13] R. Pegado, Z. Ñaupari, Y. Molina, and C. Castillo, "Radial distribution network reconfiguration for power losses reduction based on improved selective BPSO," Electric Power Systems Research, vol. 169, pp. 206-213, 2019.

[14] D. Zhang, Z. Fu, and L. Zhang, "An improved TS algorithm for loss-minimum reconfiguration in large-scale distribution systems," Electric Power Systems Research, vol. 77, no. 5-6, pp. 685-694, 2007.

[15] R. S. Rao, S. V. L. Narasimham, M. R. Raju, and A. S. Rao, "Optimal network reconfiguration of large-scale distribution system using harmony search algorithm," IEEE Transactions on Power Systems, vol. 26, no. 3, pp. 1080-1088, 2011.

[16] H. Xing and S. Hong, "Ordinal optimisation approach for complex distribution network reconfiguration," The Journal of Engineering, vol. 2019, no. 18, pp. 5055-5058, 2019.

[17] T. T. Nguyen and A. V. Truong, "Distribution network reconfiguration for power loss minimization and voltage profile improvement using cuckoo search algorithm," International Journal of Electrical Power \& Energy Systems, vol. 68, pp. 233-242, 2015.

[18] A. Mohamed Imran and M. Kowsalya, "A new power system reconfiguration scheme for power loss minimization and voltage profile enhancement using fireworks algorithm," International Journal of Electrical Power \& Energy Systems, vol. 62, pp. 312-322, 2014.

[19] A. Onlam, D. Yodphet, R. Chatthaworn, C. Surawanitkun, A. Siritaratiwat, and P. Khunkitti, "Power loss minimization and voltage stability improvement in electrical distribution system via network reconfiguration and distributed generation placement using novel adaptive shuffled frogs leaping algorithm," Energies, vol. 12, no. 3, 2019.

[20] T. T. Nguyen, T. T. Nguyen, A. V. Truong, Q. T. Nguyen, and T. A. Phung, "Multi-objective electric distribution network reconfiguration solution using runner-root algorithm," Applied Soft Computing, vol. 52, pp. 93-108, 2017.

[21] R. S. Rao, K. Ravindra, K. Satish, and S. V. L. Narasimham, "Power loss minimization in distribution system using network reconfiguration in the presence of distributed generation," IEEE Transactions on Power Systems, vol. 28, no. 1, pp. 317-325, 2013.

[22] A. Mohamed Imran, M. Kowsalya, and D. P. Kothari, “A novel integration technique for optimal network reconfiguration and distributed generation placement in power distribution networks," International Journal of Electrical Power \& Energy Systems, vol. 63, pp. 461-472, 2014.

[23] T. T. Nguyen, A. V. Truong, and T. A. Phung, "A novel method based on adaptive cuckoo search for optimal network reconfiguration and distributed generation allocation in distribution network," International Journal of Electrical Power \& Energy Systems, vol. 78, pp. 801-815, 2016.

[24] Z. Gong, Q. Chen, and K. Sun, "Novel methodology solving distribution network reconfiguration with DG placement," The Journal of Engineering, vol. 2019, no. 16, pp. 1668-1674, 2019.

[25] M. Abd El-salam, E. Beshr, and M. Eteiba, "A new hybrid technique for minimizing power losses in a distribution system by optimal sizing and siting of distributed generators with network reconfiguration," Energies, vol. 11, no. 12, p. 3351, 2018.

[26] V. V. V. S. N. Murty and A. Kumar, "Optimal DG integration and network reconfiguration in microgrid system with realistic time varying load model using hybrid optimisation," IET Smart Grid, vol. 2, no. 2, pp. 192-202, 2019.

[27] M.-Y. Cheng and D. Prayogo, "Symbiotic organisms search: a new metaheuristic optimization algorithm," Computers \& Structures, vol. 139, no. Supplement C, pp. 98-112, 2014.

[28] M.-Y. Cheng, D. Prayogo, and D.-H. Tran, "Optimizing multiple-resources leveling in multiple projects using discrete symbiotic organisms search," Journal of Computing in Civil Engineering, vol. 30, no. 3, Article ID 04015036, 2016.

[29] V. F. Yu, A. A. N. P. Redi, C.-L. Yang, E. Ruskartina, and B. Santosa, "Symbiotic organisms search and two solution representations for solving the capacitated vehicle routing problem," Applied Soft Computing, vol. 52, pp. 657-672, 2017.

[30] G. G. Tejani, V. J. Savsani, and V. K. Patel, "Adaptive symbiotic organisms search (SOS) algorithm for structural design optimization," Journal of Computational Design and Engineering, vol. 3, no. 3, pp. 226-249, 2016.

[31] D. T. T. Do and J. Lee, "A modified symbiotic organisms search (mSOS) algorithm for optimization of pin-jointed structures," Applied Soft Computing, vol. 61, pp. 683-699, 2017.

[32] A. Panda and S. Pani, "A symbiotic organisms search algorithm with adaptive penalty function to solve multi-objective constrained optimization problems," Applied Soft Computing, vol. 46, pp. 344-360, 2016.

[33] S. Duman, "Symbiotic organisms search algorithm for optimal power flow problem based on valve-point effect and prohibited zones," Neural Computing and Applications, vol. 28, no. 11, pp. 3571-3585, 2017.

[34] H. Kamankesh, V. G. Agelidis, and A. Kavousi-Fard, "Optimal scheduling of renewable micro-grids considering plugin hybrid electric vehicle charging demand," Energy, vol. 100, pp. 285-297, 2016.

[35] S. Verma, S. Saha, and V. Mukherjee, "A novel symbiotic organisms search algorithm for congestion management in deregulated environment," Journal of Experimental \& Theoretical Artificial Intelligence, vol. 29, no. 1, pp. 59-79, 2017.

[36] S. Saha and V. Mukherjee, "Optimal placement and sizing of DGs in RDS using chaos embedded SOS algorithm," IET Generation, Transmission \& Distribution, vol. 10, no. 14, pp. 3671-3680, 2016.

[37] D.-H. Tran, M.-Y. Cheng, and D. Prayogo, "A novel multiple objective symbiotic organisms search (MOSOS) for time-costlabor utilization tradeoff problem," Knowledge-Based Systems, vol. 94, pp. 132-145, 2016.

[38] T. T. Tran, K. H. Truong, and D. N. Vo, "Stochastic fractal search algorithm for reconfiguration of distribution networks 
with distributed generations," Ain Shams Engineering Journal, vol. 11, no. 2, pp. 389-407, 2020.

[39] E. Dolatdar, S. Soleymani, and B. Mozafari, "A new distribution network reconfiguration approach using a tree model," World Academy of Science, Engineering and Technology, vol. 58, no. 34, p. 1186, 2009.

[40] A. Y. Abdelaziz, F. M. Mohamed, S. F. Mekhamer, and M. A. L. Badr, "Distribution system reconfiguration using a modified tabu search algorithm," Electric Power Systems Research, vol. 80, no. 8, pp. 943-953, 2010.

[41] M. E. Baran and F. F. Wu, "Network reconfiguration in distribution systems for loss reduction and load balancing," IEEE Transactions on Power Delivery, vol. 4, no. 2, pp. 14011407, 1989.

[42] A. Bayat, A. Bagheri, and R. Noroozian, "Optimal siting and sizing of distributed generation accompanied by reconfiguration of distribution networks for maximum loss reduction by using a new UVDA-based heuristic method," International Journal of Electrical Power \& Energy Systems, vol. 77, pp. 360-371, 2016.

[43] R. Rajaram, K. Sathish Kumar, and N. Rajasekar, "Power system reconfiguration in a radial distribution network for reducing losses and to improve voltage profile using modified plant growth simulation algorithm with distributed generation (DG)," Energy Reports, vol. 1, pp. 116-122, 2015.

[44] H.-D. Chiang and R. Jean-Jumeau, "Optimal network reconfigurations in distribution systems I. A new formulation and a solution methodology," IEEE Transactions on Power Delivery, vol. 5, no. 4, pp. 1902-1909, 1990.

[45] S. Ching-Tzong and L. Chu-Sheng, "Network reconfiguration of distribution systems using improved mixed-integer hybrid differential evolution," IEEE Transactions on Power Delivery, vol. 18, no. 3, pp. 1022-1027, 2003.

[46] A. Ahuja, S. Das, and A. Pahwa, "An AIS-ACO hybrid approach for multi-objective distribution system reconfiguration," IEEE Transactions on Power Systems, vol. 22, no. 3, pp. 1101-1111, 2007.

[47] C. Wang and H. Z. Cheng, "Optimization of network configuration in large distribution systems using plant growth simulation algorithm," IEEE Transactions on Power Systems, vol. 23, no. 1, pp. 119-126, 2008.

[48] C.-T. Su, C.-F. Chang, and J.-P. Chiou, "Distribution network reconfiguration for loss reduction by ant colony search algorithm," Electric Power Systems Research, vol. 75, no. 2-3, pp. 190-199, 2005. 